\title{
Modes d'habiter à Pompéi à l'époque républicaine : diffusion et utilisation du type de la maison à atrium testudinatum
}

La campagne 2020 - recherches en I 16, 5

Dora D'Auria, Pascale Ballet et Marcella Leone

\section{OpenEdition}

Journals

Édition électronique

URL : https://journals.openedition.org/baefe/2126

DOI : $10.4000 /$ baefe. 2126

ISSN : 2732-687X

Éditeur

ResEFE

Référence électronique

Dora D'Auria, Pascale Ballet, Marcella Leone, « Modes d'habiter à Pompéi à l'époque républicaine diffusion et utilisation du type de la maison à atrium testudinatum » [notice archéologique], Bulletin archéologique des Écoles françaises à l'étranger [En ligne], Italie, mis en ligne le 22 mai 2021, consulté le 24 mai 2021. URL : http://journals.openedition.org/baefe/2126; DOI : https://doi.org/10.4000/baefe. 2126

Ce document a été généré automatiquement le 24 mai 2021.

Le Bulletin archéologique des Écoles françaises à l'étranger est mise à disposition selon les termes de la Licence Creative Commons Attribution - Pas d'Utilisation Commerciale - Pas de Modification 4.0 International. 


\section{Modes d'habiter à Pompéi à l'époque républicaine : diffusion et utilisation du type de la maison à atrium testudinatum}

La campagne 2020 - recherches en I 16, 5

Dora D'Auria, Pascale Ballet et Marcella Leone

\section{NOTE DE L'AUTEUR}

Date précise de l'opération : 7 septembre-2 octobre 2020

Autorité nationale présente : Parco archeologico di Pompei - MiC

Numéro de mission : Concession MIBACT DGABAP Prot. n. 0019331 du 26/06/2020

Composition de l'équipe de terrain : Pascale Ballet, archéologue (Université Paris Nanterre) ; Guilhem Chapelin, architecte (Centre Jean Bérard, USR 3133, CNRS-EFR); Dora D'Auria, archéologue (Università degli studi di Napoli L'Orientale) ; Federica Giacobello, archéologue (Università degli studi di Milano Statale) ; Marcella Leone, archéologue - céramologue (Centre Jean Bérard, USR 3133, CNRS-EFR).

Partenariats institutionnels : CJB, Université Paris Nanterre, Università degli studi di Napoli L'Orientale

Établissement éditeur : $\mathrm{CJB}$

Établissements porteurs de l'opération : CJB, Université Paris-Nanterre

Remerciements : Nous remercions le directeur du Parco Archeologico di Pompei, Massimo Osanna, d'avoir accueilli favorablement notre projet de recherche et les fonctionnaires Maria Laura Iadanza et Luana Toniolo pour leur appui. Nous souhaitons également exprimer notre gratitude à toute l'équipe du Centre Jean Bérard, et tout particulièrement à la directrice Claude Pouzadoux et à la directrice adjointe Priscilla Munzi, pour leur précieux soutien. 
Données scientifiques produites :

https://centrejeanberard.cnrs.fr/spip.php?article400\&lang=fr

Chroniques de l'EFR :

http://journals.openedition.org/cefr/4796

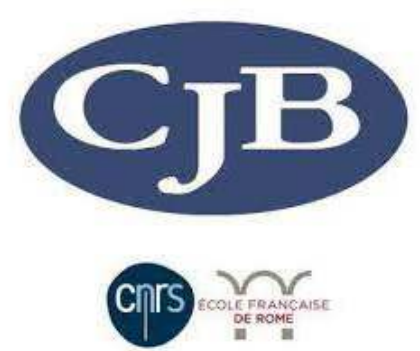

\section{Introduction}

1 Le projet, initié sur le terrain en juillet 2019², est centré sur l'habitat urbain de Pompéi, en particulier sur les maisons à atrium testudinatum, et fait l'objet d'une double approche, architecturale et sociologique.

2 Le projet, porté par l'université Paris Nanterre, est inscrit dans les programmes du Centre Jean Bérard (USR 3133, CNRS-EFR), notamment dans l'axe 2 « Espaces, économie et artisanat dans les cités vésuviennes ", sous-axe " Organisation et gestion des espaces urbains et périurbains » et fait partie des dossiers de la Mission archéologique «Italie du Sud» (Ministère de l'Europe et des Affaires étrangères, 2020-2023). Le projet est associé à l'Università degli studi di Napoli L'Orientale. En 2020, le Ministero per i Beni e le Attività Culturali e del Turismo, désormais Ministero della Cultura, nous a accordé une concession de recherche et de fouilles archéologiques (Concession Prot. MIBACT DGABAP $0019331 \mathrm{du}$ 26/06/2020). Enfin, le projet est inscrit dans les axes de recherche de l'équipe ESPRI (Espaces, pratiques sociales et images dans le monde grec et romain), l'une des composantes de l'UMR 7041/ArScAn, dont les terrains inscrits dans l'espace méditerranéen (Grèce centrale, Italie, Égypte du nord) permettent, par leur diversité, de croiser les modes d'habiter.

Il s'agit d'explorer un type d'habitat de l'époque républicaine sous l'angle de ses spécificités architecturales, indicateur « de modes d'habiter » et expression de l'une des composantes de la population urbaine. Conduisant donc à analyser les manières d'occuper l'espace de vie privé(e), le projet s'inscrit dans une étude de la société, puisque les caractéristiques architecturales et le décor associé permettent de définir un certain niveau de vie des occupants. Le projet concerne les mutations qu'ont connues ces habitations précoces et en appréhende les réaménagements de leur fondation jusqu'en 79. Les cas sélectionnés, les unités VI 11, 11-12/7 et I 16, 5-7, ont été choisis dans deux secteurs de la ville, les Regiones I et VI, éloignés topographiquement et qui ont eu un développement urbain et social différent. 


\section{Actions menées lors de la campagne de septembre 2020}

En 2020, les activités de recherche ont pu se dérouler sur le terrain, du 7 septembre au 2 octobre. Elles ont concerné la partie méridionale de l'îlot I 16, secteur dans lequel sont situés deux des cas d'étude de ce projet, les maisons à atrium testudinatum n. 5 et 6 (fig. 1). Après la première entreprise menée en 2019 dans la maison de la Regio VI la VI 11, 11-12/7 - qui a permis de restituer les phases de constructions d'une partie de la demeure, nous avons opté, dans le cadre de la programmation, d'élargir cette année l'étude des habitats à atrium testudinatum aux exemples initialement sélectionnés de la Regio I. En effet, la crise sanitaire et la nécessité de respecter les mesures restrictives mises en place pour lutter contre la diffusion de l'épidémie de Covid-19 nous ont contraints à limiter le nombre des participants à la mission et à renoncer à l'exécution de sondages de fouilles dans la maison VI 11, 11-12/7, afin de garantir au maximum la distanciation physique parmi les participants à la mission ${ }^{2}$. Par conséquent, ne pouvant réunir une équipe de fouilleurs afin de procéder à l'étude fine de cette habitation, il nous a semblé opportun de nous orienter vers l'analyse architecturale et stratigraphique d'une maison du secteur méridional de l'insula I16, inscrite au programme.

Fig. 1. Plan de I 16, 5-7.

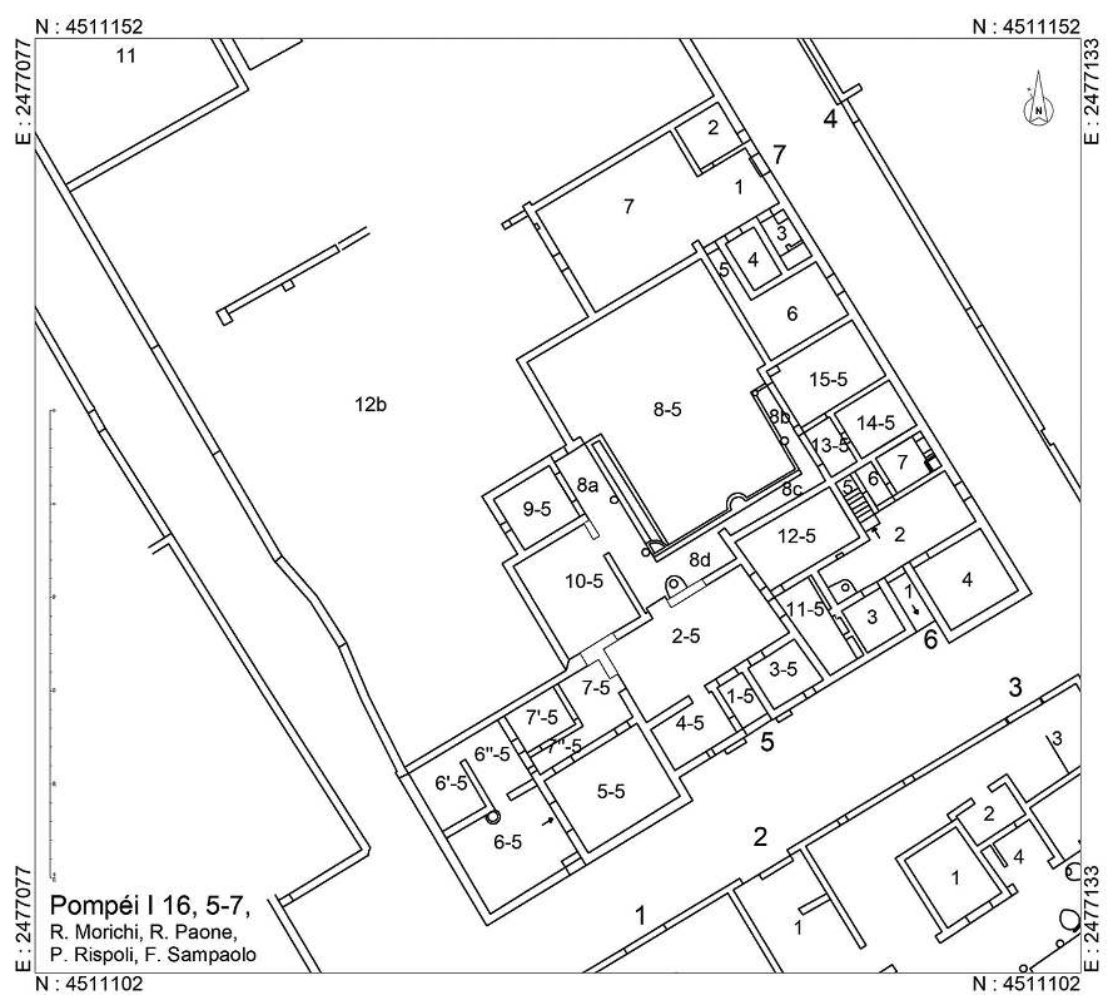

D’après R. Morichi, R. Paone, P. Rispoli, F. Sampaolo.

5 Les recherches sur le terrain ont donc été consacrées à l'analyse stratigraphique et au relevé par photogrammétrie des structures in situ de I 16,5-7, ainsi qu'au rangement des mobiliers retrouvés pendant le dégagement d'une partie de la maison n. 5 et stockés à son intérieur. Les données récoltées ont été enregistrées dans une base de 
données ${ }^{3}$ structurée, en partant des modèles élaborés par l'Istituto Centrale per il Catalogo e la Documentazione (ICCD) ${ }^{4}$, institut chargé de la documentation et du classement du patrimoine en Italie. Les fiches de l'ICCD ont néanmoins été modifiées pour les adapter aux particularités des édifices pompéiens et afin de faciliter leur utilisation pour les utilisateurs moins à l'aise avec l'analyse du bâti et les particularités de l'architecture pompéienne. On a inséré, par exemple, des zones de listes déroulantes et ajouté des champs destinés au matrix et à un croquis de l'unité analysée, afin de faciliter la saisie et l'interprétation des données 5 .

Par ailleurs, une recherche dans les archives scientifiques du Parco Archeologico di Pompei a été menée, afin de retrouver les données concernant le dégagement des édifices, notre objet d'étude, qui ont été mis au jour en deux temps, à la fin des années 1950 et en 1992 (fig. 2). À cause d'une indisponibilité de la section des archives concernant les activités de la fin du $\mathrm{xx}^{\mathrm{e}}$ siècle, cette année, nos recherches se sont concentrées sur les journaux de fouille des années 1950, analysant les versions soit manuscrite ${ }^{6}$, soit dactylographiée pour trois années, 1957-19597.

Fig. 2. Plan de l'insula I 16 après les fouilles des années 1950.

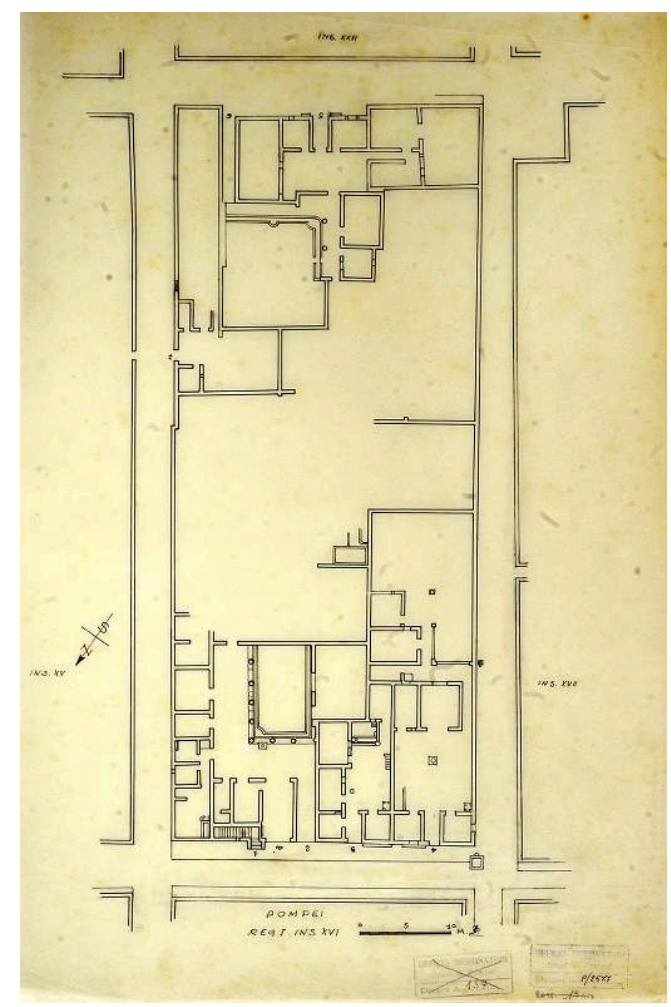

La portion sud-est de l'îlot, qui sera dégagée en 1992, était encore ensevelie.

Archives Parco Archeologico di Pompei, sur concession du MiC - PA Pompei. Tous droits réservés.

\section{Présentation de la maison I 16, 5}

Le secteur méridional de l'insula I 16 se compose de trois unités : deux maisons à atrium testudinatum, n. 5 et 6 , accessibles depuis la rue qui borde lîlot au sud, la via della Palestra, et un édifice, n. 7, dont la fonction est à préciser, auquel on accède par une porte donnant sur la rue qui longe l'îlot à est, le Vico della Nave Europa. Les deux 
maisons à atrium testudinatum ont été probablement édifiées - comme les techniques de construction semblent le suggérer - à des époques diverses au courant des $\mathrm{III}^{\mathrm{e}}$ et $\mathrm{II}^{\mathrm{e}}$ siècles av. J.-C. et témoignent de niveaux de richesse hétérogènes et différents de ceux de la domus VI 11,11-12/7, ce qui justifie le cadre de notre examen portant sur un échantillon varié. L'objectif de nos recherches dans ce secteur est d'obtenir des données sur les différentes formes et les modes d'utilisation du type d'habitat, objet de notre étude, et de restituer l'histoire de la construction des bâtiments pris en compte, de la fondation jusqu'à l'ensevelissement en 79 apr. J.-C.

Les activités sur le terrain, menées pendant la dernière campagne, se sont concentrées sur la zone nord-est de la maison n. 5. Cette demeure, à l'époque de l'éruption, est composée de trois secteurs. Le premier auquel on a accès de l'extérieur est un atrium testudinatum qui, en 79, est en communication avec plusieurs salles. Sur le côté méridional, s'ouvrent deux pièces (3 et 4) qui flanquent le couloir d'entrée, chacune d'entre elles étant pourvue d'une fenêtre donnant sur la rue. Dans la pièce (4), des peintures du $\mathrm{I}^{\mathrm{er}}$ style sont conservées à la base des murs nord, ouest et sud ${ }^{8}$. Sur le côté oriental, deux portes donnent accès à deux salles communiquant entre elles, les pièces (11) et (12). Dans la première (11), une distinction entre antichambre et alcôve est encore perceptible grâce à la décoration pariétale ${ }^{9}$ qui remonte à la période du $\mathrm{I}^{\mathrm{er}}$ style (fig. 3) et qui, dans l'antichambre, est caractérisée par une peinture à schéma décoratif simple, constitué d'un socle jaune et des zones moyenne et supérieure blanches et plates, terminant au sommet avec une bande en relief. En revanche, l'alcôve est dotée d'un décor plus riche, du I ${ }^{\text {er }}$ style, avec socle rouge, orthostates verts et zone supérieure dans un mauvais état de conservation. L'une des particularités de cette pièce concerne le mur est qui, en 79, est entièrement occupé par des renfoncements de forme et de dimensions variés, le renfoncement central étant utilisé comme placard. Une porte dans le mur nord met en communication cette pièce avec le salon (12). Bien que les peintures soient ici (12) dans un mauvais état de conservation, on reconnaît aisément deux phases décoratives: la première est constituée d'un bandeau et de corniches en stuc appartenant à la zone supérieure d'un schéma de $\mathrm{I}^{\mathrm{er}}$ style; pour la deuxième, de III ${ }^{\mathrm{e}}$ style, on distingue encore un socle à fond noir, enrichi d'un motif de jardin, et une zone moyenne séparée en trois panneaux, dont il ne reste que peu d'éléments. 
Fig. 3. La pièce (11) vue du nord.

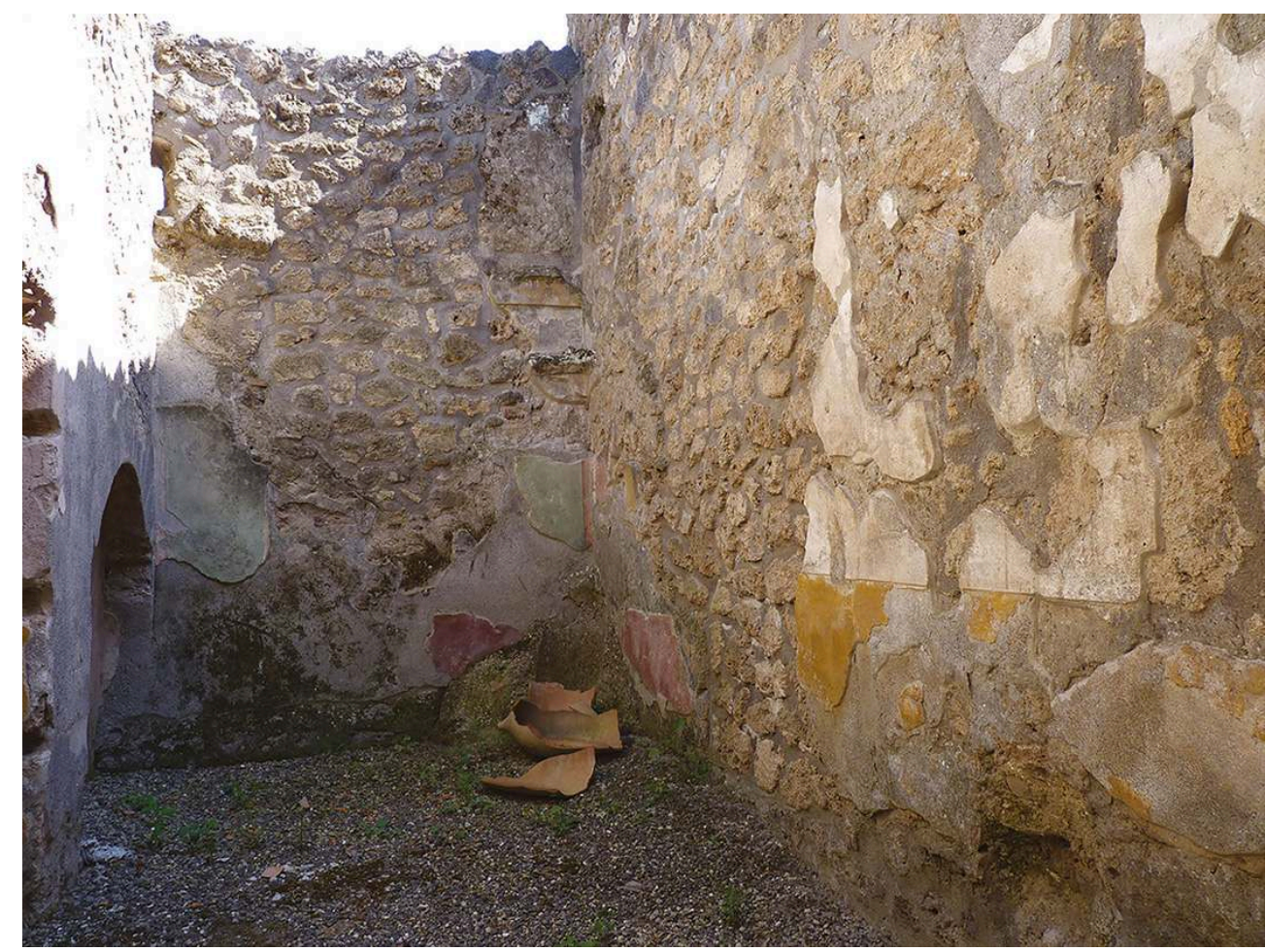

D. D'Auria, sur concession du MiC - PA Pompei. Tous droits réservés

9 Sur le côté occidental de l'atrium est située une porte séparant cet espace d'un secteur occupé par des pièces de service et peut-être de production, dont la fonction pourra être précisée lors de nos recherches ultérieures. Dans cette zone, deux larges espaces, (5) et (6), sont reconnaissables sur lesquels s'ouvrent des pièces de plus petites dimensions. L'espace n. 6 , situé plus à l'intérieur et pourvu de la bouche d'une réserve d'eau, est défini comme « jardin » dans les journaux de fouille des années $1950^{10}$.

Enfin, depuis un passage situé dans le mur nord de l'atrium, on a accès à un jardin à portiques (8) ; sur ses côtés est et ouest s'ouvrent des salles à caractère résidentiel. Les portiques ne ceinturent pas complétement cet espace, mais ils sont situés exclusivement en correspondance avec l'entrée des pièces. Le jardin (8) est séparé des portiques par un muret de faible hauteur, auquel est associé un système de récolte des eaux de pluie. Sur le côté occidental, ce système est constitué d'une sorte de bassin rectangulaire, qui, situé aux pieds des colonnes, prend la place du caniveau habituel récoltant les eaux tombant du toit. Le long du couloir qui, au sud du jardin, met en communication les deux portiques est situé, en revanche, un caniveau maçonné et revêtu de mortier de tuileau (fig. 4), assurant la récupération de l'eau tombant du pan de toit couvrant le salon (12). L'eau est ensuite canalisée dans un bassin situé près de la colonne méridionale du portique ouest (8a). Sur ce dernier, s'ouvrent deux salles (9) et (10). La première (9) est revêtue d'un sol en mortier de tuileau rouge, dont seulement une partie est actuellement dégagée, l'espace correspondant au seuil d'entrée comprenant un motif à quadrillage losangé (fig. 5). Ce pavement est caractéristique de l'époque du $\mathrm{I}^{\mathrm{er}}$ style, période durant laquelle cette pièce formait une exèdre largement ouverte sur le jardin. À l'extrémité nord du portique (8a) à l'époque de l'éruption, une porte met en communication la maison n. 5 avec un large hortus, avec lequel, au nord, 
communique aussi un autre bâtiment, le n. 2. Ce dernier est un édifice à la planimétrie singulière, organisé autour d'un péristyle à chapiteaux cubiques ${ }^{11}$ et dont la destination est encore à préciser.

Fig. 4. Le caniveau en mortier de tuileau qui longe l'espace (8 c-d) au sud de l'hortus.

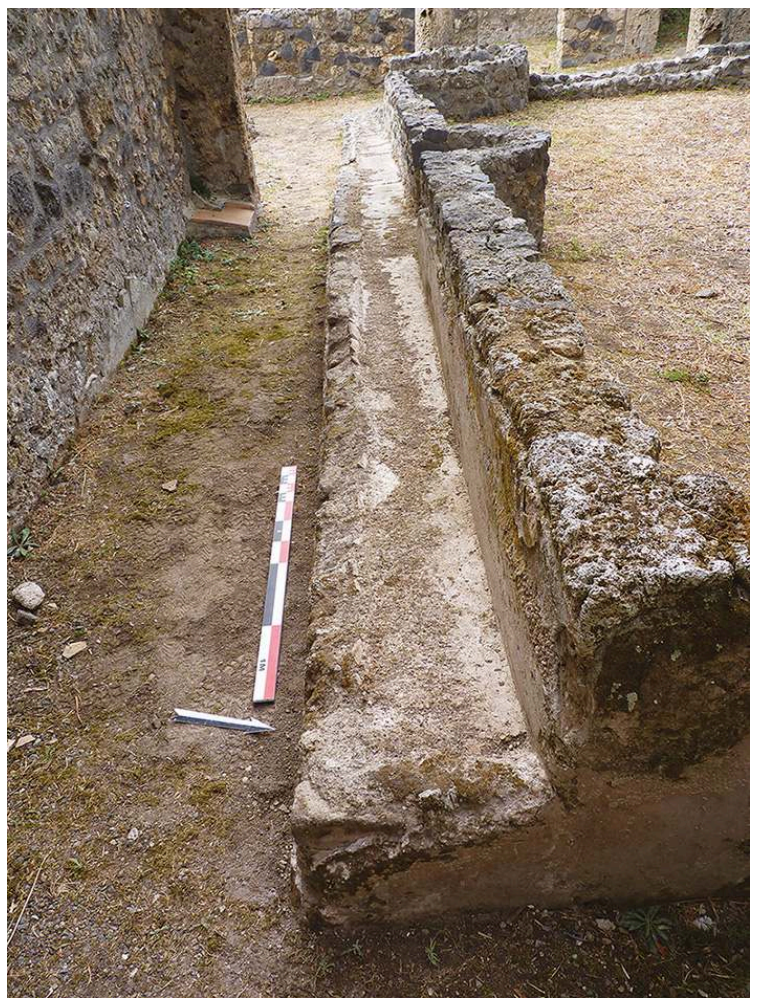

D. D’Auria, sur concession du MiC - PA Pompei. Tous droits réservés.

Fig. 5. Sol de la pièce (9).

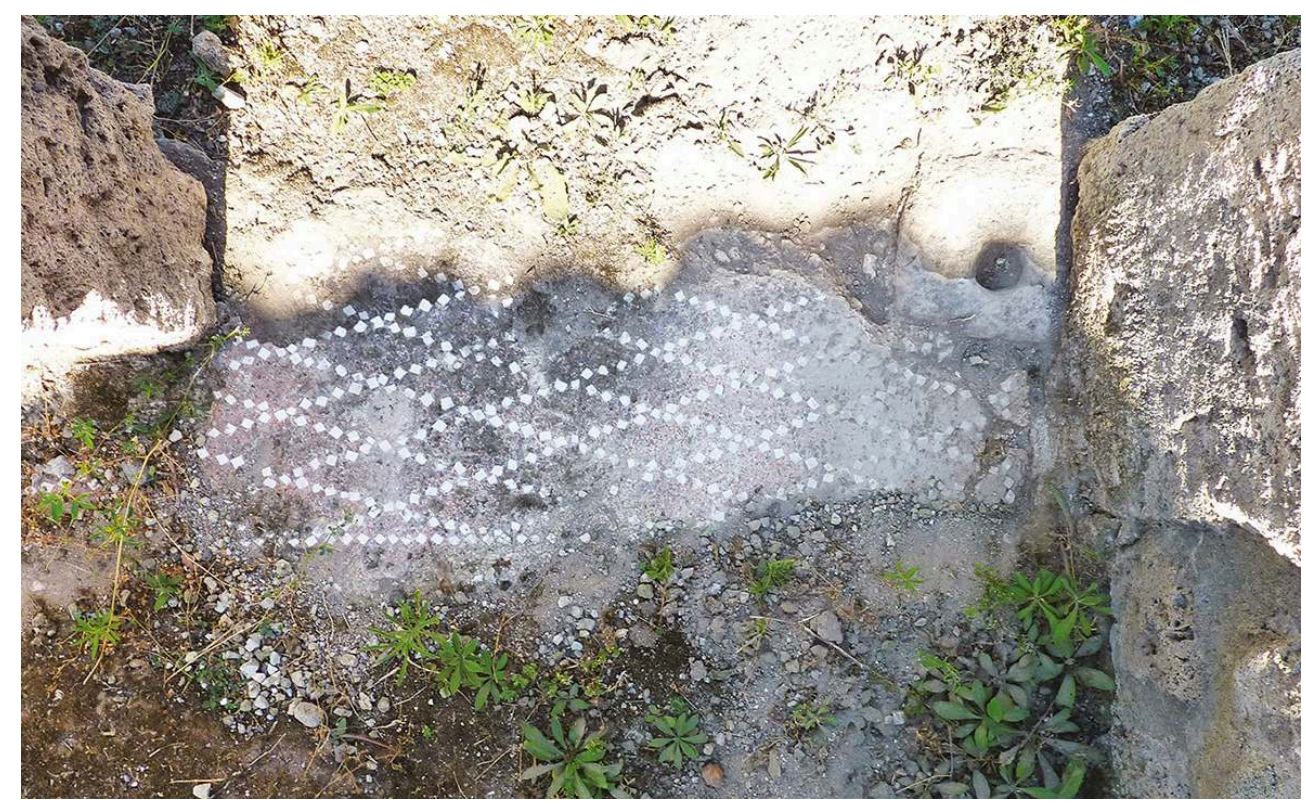

D. D’Auria, sur concession du MiC - PA Pompei. Tous droits réservés. 


\section{Le secteur analysé au courant de la campagne de 2020}

11 Sur le portique est (8b), s'ouvre une porte donnant accès à un espace de dégagement (13) qui conduit à l'intérieur d'une suite comprenant deux pièces, le salon (15) et le cubiculum (14) ${ }^{12}$. Cette année, cet espace a fait l'objet d'une étude approfondie grâce à une analyse stratigraphique des structures qui le composent. L'examen a permis de restituer l'histoire de construction de ce secteur, dans laquelle il a été possible de distinguer cinq phases. Un bilan préliminaire de cette analyse est présenté ci-dessous.

Les phases de construction du secteur de la maison I 16, 5 occupé par les pièces (13), (14) et (15)

\section{Phase 1 - Un espace ouvert}

Dans un premier temps, ce secteur était un espace non édifié, sur lequel s'ouvraient les fenêtres du mur sud (mur 1 ; fig. 8) de la pièce (6) de l'édifice 7. Ce dernier, à cette époque, devait être plus développé vers l'ouest par rapport aux phases suivantes et devait occuper une partie du jardin à portiques (8) de la future domus 5.

Le mur périmétral méridional de l'édifice 7 (mur 1) est édifié en grand appareil quadrangulaire constitué d'une alternance d'assises de parpaings en carreaux et en panneresse (fig. 6) ; la chaîne d'angle comprend une alternance d'assises de parpaings en boutisse et en panneresse. Pendant cette première phase, le mur était pourvu de deux fenêtres orientées en direction de l'espace plus tard occupé par des pièces (13), (14) et (15), lequel devait donc être ouvert (fig. 7). La technique de construction des murs de l'édifice 7 suggère un horizon chronologique d'époque médio-samnite pour sa fondation, à dater probablement du III ${ }^{\mathrm{e}}$ siècle av. J.-C. 
Fig. 6. Photogrammétrie de la paroi nord du salon (15), chronologie relative des US identifiées.

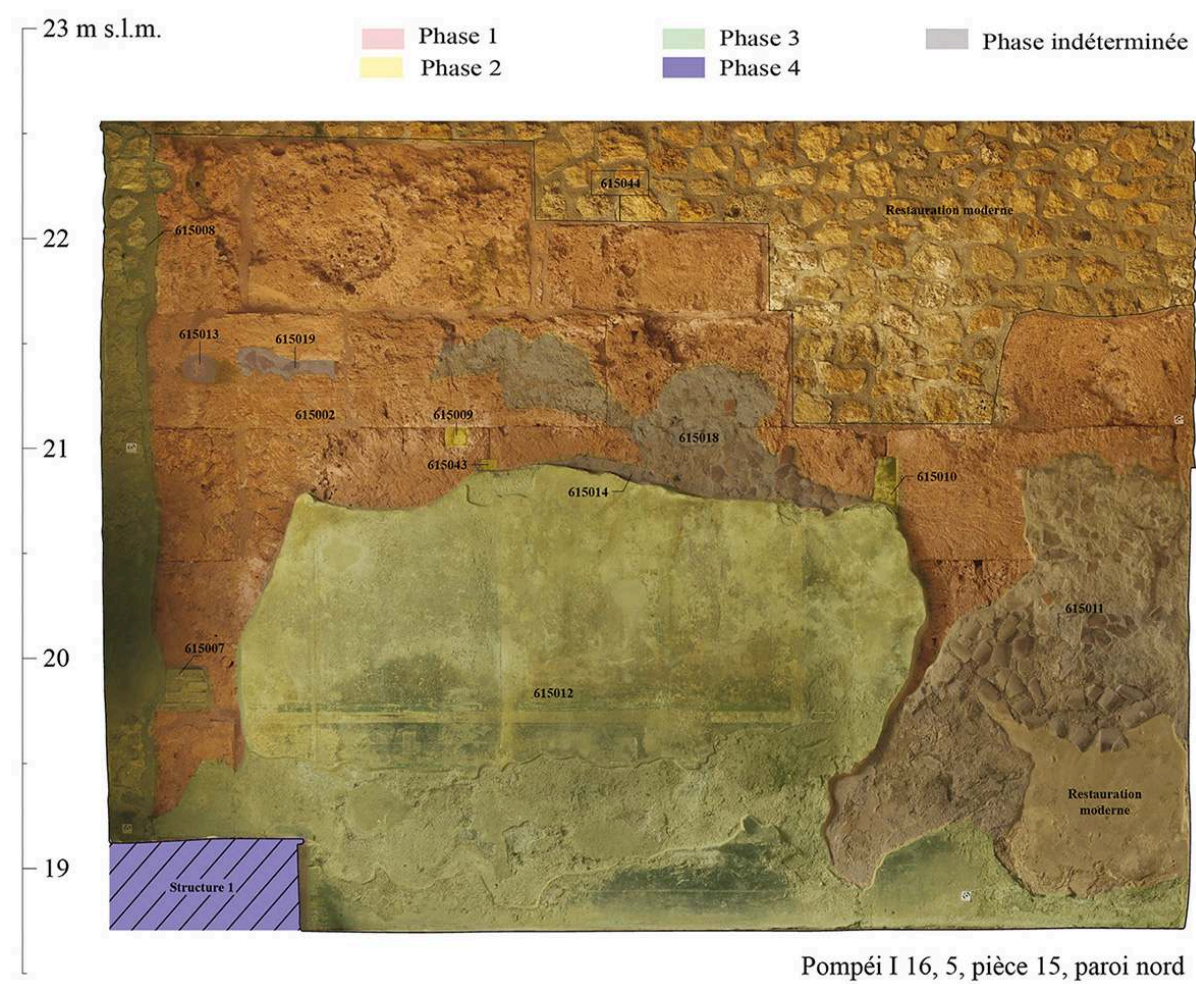

G. Chaplin, D. D'Auria, sur concession du MiC - PA Pompei. Tous droits réservés.

Fig. 7. Photogrammétrie de la paroi sud de la pièce (6) de I 16, 7, avec indication des fenêtres bouchées.

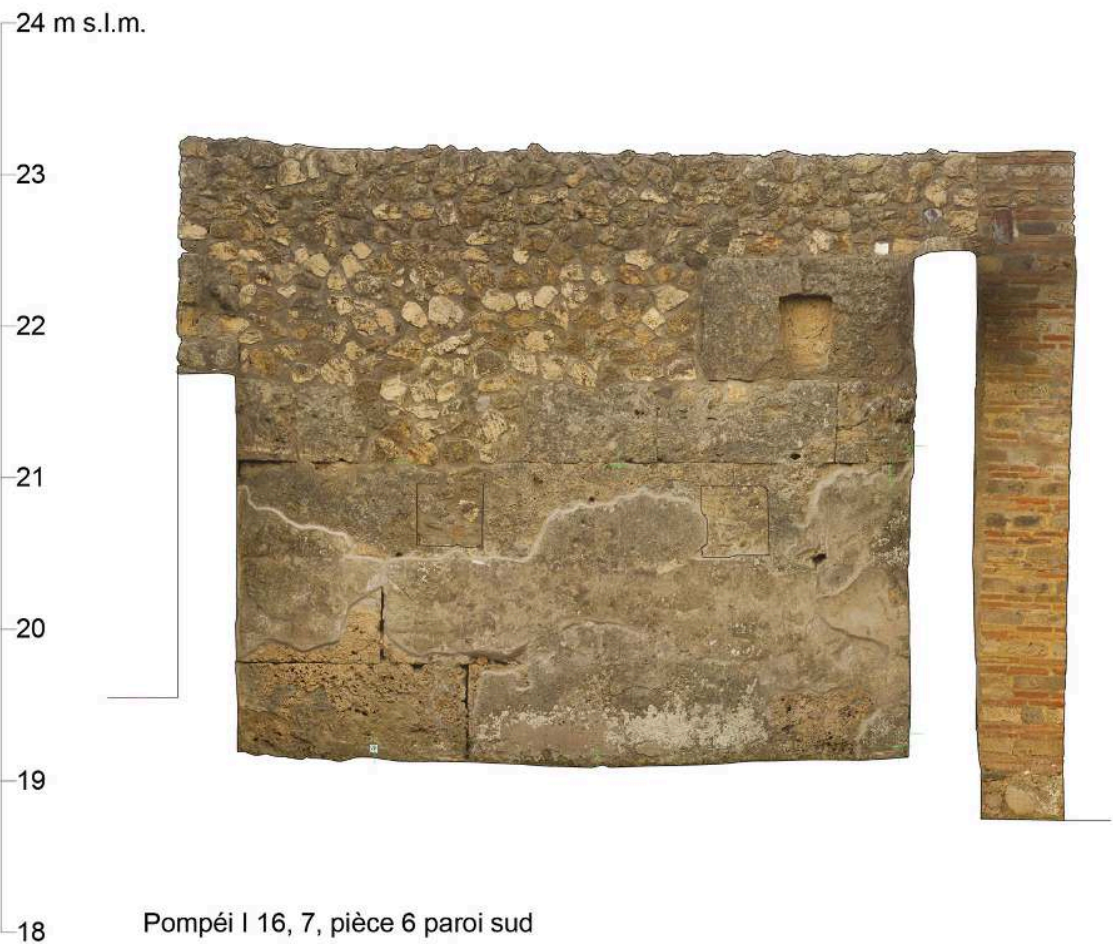

G. Chaplin, sur concession du MiC - PA Pompei. Tous droits réservés. 


\section{Phase 2 - Construction d'une salle résidentielle (13-14-15)}

Plus tard, dans ce secteur prend place un salon élégant $\left(36 \mathrm{~m}^{2}\right)$ ouvrant largement vers l'ouest sur un espace qu'occupait très vraisemblablement un jardin. Conjointement à l'aménagement de cet espace couvert, auparavant libre de constructions, les deux fenêtres du mur en grand appareil quadrangulaire (mur 1) appartenant à l'édifice n. 7 sont bouchées, en utilisant de l'opus incertum de lave (fig. 6, UC 615010 et 615043).

La nouvelle pièce, fermée au nord par le mur périmétral sud de l'édifice 7, est délimitée par de nouveaux murs (murs 2 et 3) bâtis en opus incertum de calcaire. Le mur méridional (mur 3) semble être une reconstruction de l'ancienne limite nord de la maison I 16, $6^{13}$. Le côté occidental du salon est à cette époque fermé par un mur qui sera détruit par la suite et qui est caractérisé par une large ouverture occupant la plupart de sa longueur.

De la décoration de ce salon, correspondant à l'emplacement des futures pièces décor riche et complexe (fig. 8). Il est constitué de motifs géométriques caractéristiques des sols $\mathrm{du} \mathrm{II}^{\mathrm{e}}$ siècle av. J.-C. ${ }^{14}$, réalisés en utilisant des tesselles blanches de calcaire, ici enrichis de pierres colorées, ce qui constitue un élément particulier. L'espace correspondant au seuil est orné d'un quadrillage losangé, une pierre colorée étant insérée au milieu de chaque élément ${ }^{15}$; la bande de partition qui sépare le seuil du tapis est constituée de pierres colorées de forme oblongue disposées en arêtes de poisson aux deux côtés d'une ligne formée de tesselles blanches ${ }^{16}$ (fig. 9). Le tapis est décoré d'un semis irrégulier et dense d'écailles polychromes ${ }^{17}$ (fig. 10) et est encadré par une bande avec le motif du méandre de svastikas et de carrés avec pierre colorée au centre (fig. 11). Autour du tapis, l'espace en forme de $\Pi$ est décoré d'un motif plus simple, constitué d'un semis irrégulier d'écailles polychromes moins dense que le précédent et formé d'écailles de plus petites dimensions ${ }^{18}$ (fig. 12). Les couleurs des pierres utilisées pour le décor de ce pavement sont jaune, vert, rouge et noir. Dans les losanges et le tapis central, le blanc est aussi utilisé, tandis que dans les bandes latérales, le rouge est absent. Les tesselles blanches de calcaire utilisées pour réaliser les motifs ont une forme quasiment cubique, de coupe irrégulière ${ }^{19}$. 
Fig. 8. Photogrammétrie du pavement des pièces (13), (14) et (15).

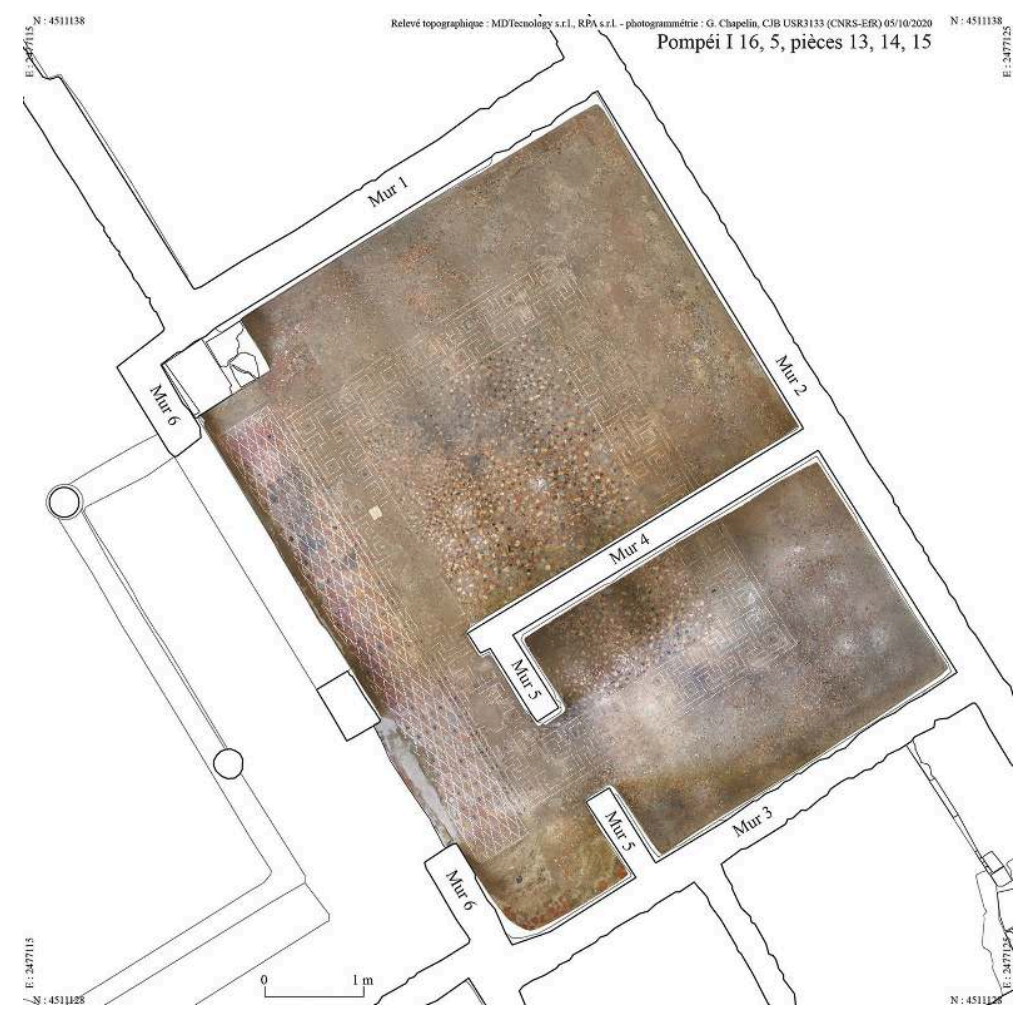

G. Chaplin, sur concession du MiC - PA Pompei. Tous droits réservés.

Fig. 9. Sol du salon (15), détail de la ligne de partition entre le seuil et le tapis.

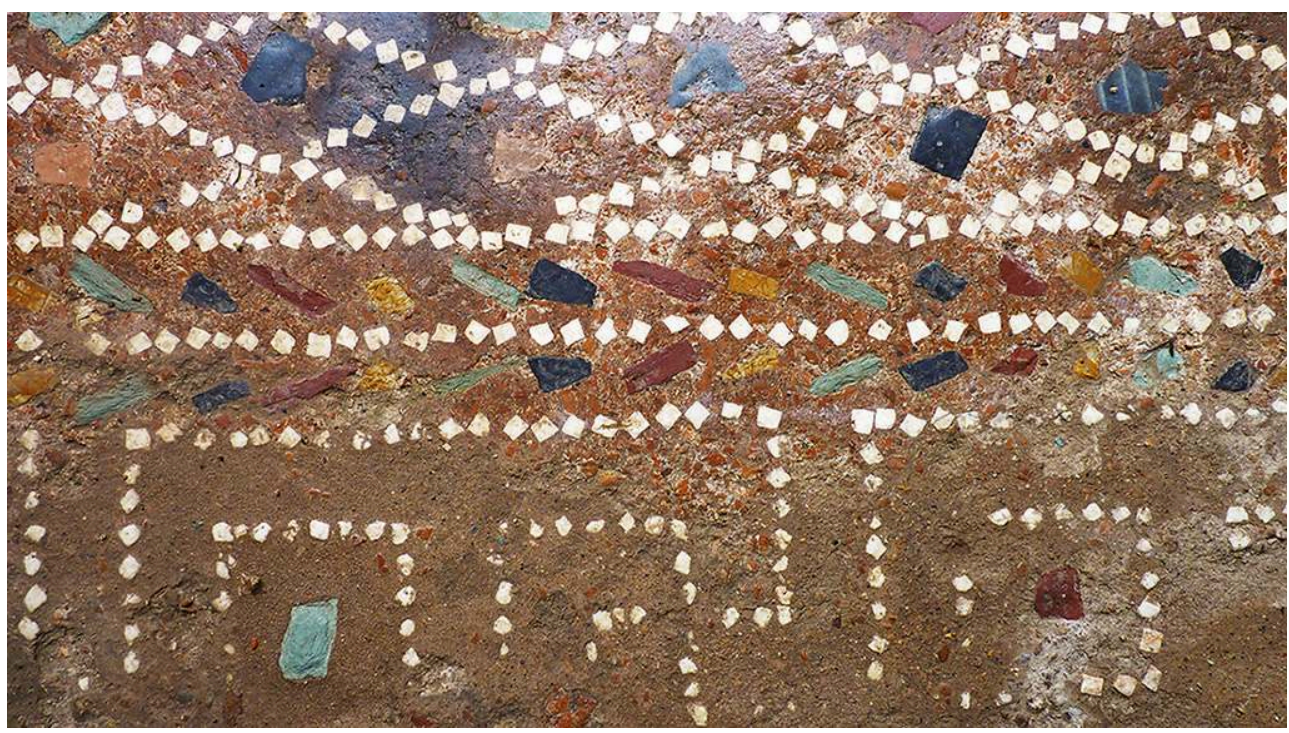

D. D’Auria, sur concession du MiC - PA Pompei. Tous droits réservés. 
Fig. 10. Sol du salon (15), détail du tapis.

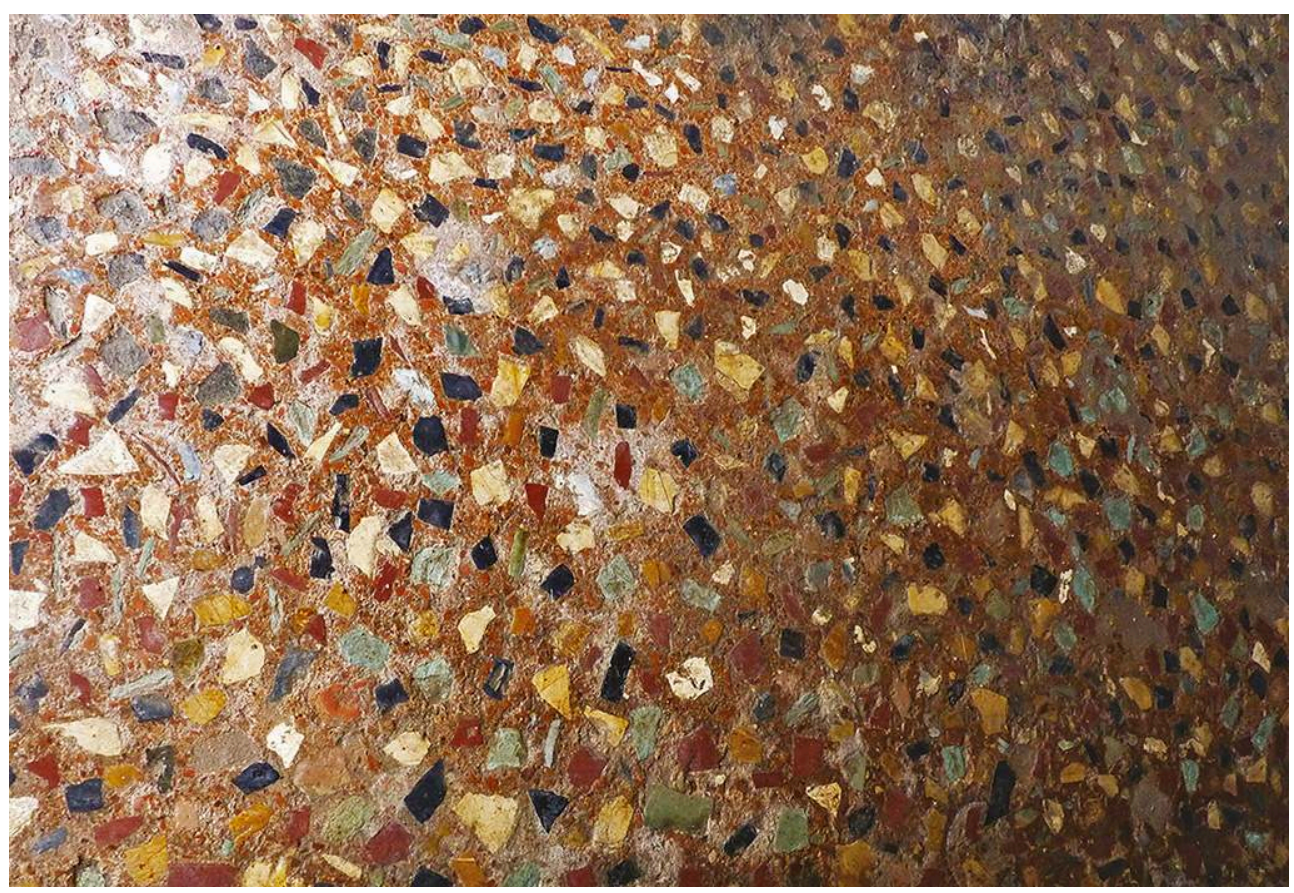

D. D'Auria, sur concession du MiC - PA Pompei. Tous droits réservés.

Fig. 11. Sol du salon (15), détail de la bande du tapis.

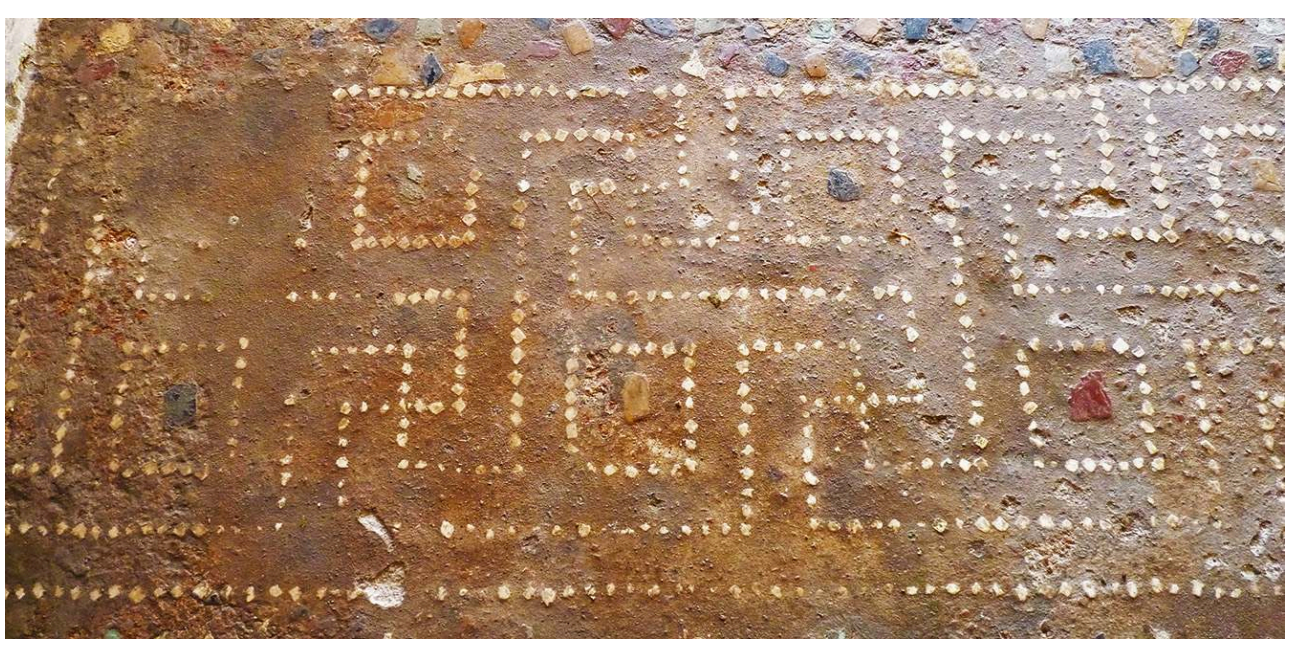

D. D'Auria, sur concession du MiC - PA Pompei. Tous droits réservés. 
Fig. 12. Pièce (14), sol.

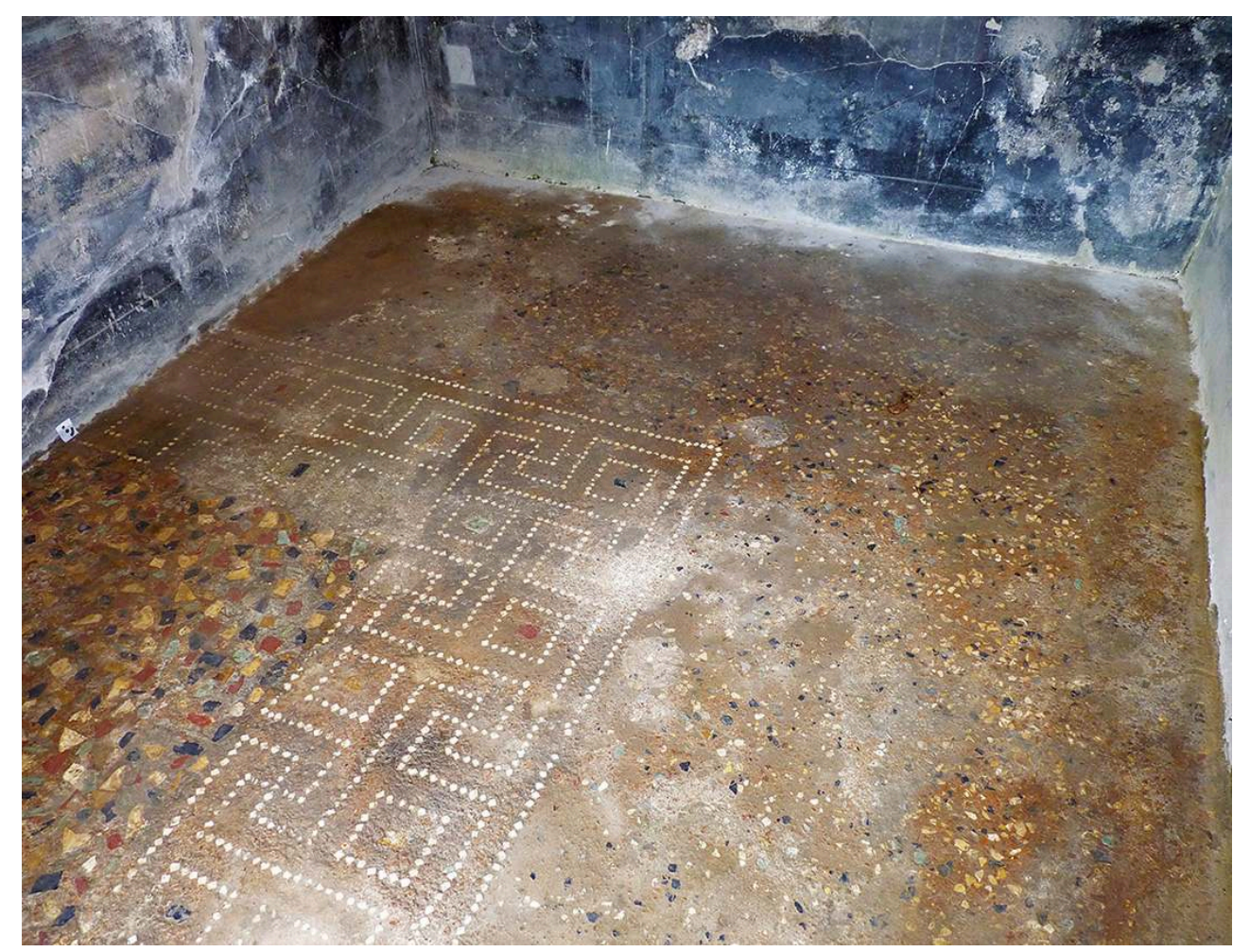

D. D'Auria, sur concession du MiC - PA Pompei. Tous droits réservés.

\section{Phase 3 - Division de l'espace en trois pièces}

À une phase plus récente, à faire probablement remonter au début du $\mathrm{I}^{\mathrm{er}}$ siècle apr. J.-C. comme cela est suggéré par le style des peintures, l'ancien salon est divisé en trois pièces : l'oecus (15), la pièce (14) et l'espace de dégagement (13). Ce dernier constitue une sorte d'antichambre de l'ensemble, le mettant en communication avec le jardin (8) et faisant de liaison entre les deux salles, lesquelles n'avaient pas de portes avec un système de fermeture fixe.

18 La réorganisation de l'espace initial en trois nouvelles pièces est effectuée grâce à la construction de deux murs (mur 4 et 5) appuyés sur l'ancien pavement en mortier de tuileau, lequel continue à constituer le revêtement du sol de l'ensemble. La technique de construction des nouveaux murs n'est quasiment pas visible, à cause du revêtement d'enduit et des lacunes. Dans les parties visibles, plutôt réduites, il semble possible de reconnaitre un opus incertum de calcaire. Ces deux murs présentent des ouvertures. Le mur 4 est percé d'une porte près de sa limite ouest qui met en communication l'espace (13) avec le salon (15). Elle a un seul montant à l'est, édifié en opus vittatum, et sans seuil, ce qui indique qu'elle n'était pas équipée d'un système de fermeture fixe. Une deuxième porte est située dans le mur 5 et met en communication l'espace (13) avec la pièce (14). Elle présente les mêmes caractéristiques que l'autre ouverture, étant pourvue de montants, deux cette fois-ci, en opus vittatum et dépourvue de seuil.

19 Le réaménagement de l'espace (13-14-15) est complété par la réfection du mur ouest de l'ancien salon qui est reconstruit (mur 6) une vingtaine de centimètres plus à l'ouest. Cette modification comporte l'agrandissement du mur 1 (fig. 6, UC 615008). 
En complétement de ces travaux, les parois des pièces (14) et (15) sont revêtues d'un enduit peint à décor du $\mathrm{III}^{\mathrm{e}}$ style. Les peintures ne recouvrent pas la totalité de la surface de la paroi : en effet, les montants des portes ne présentent pas d'enduit peint. C'est le cas de l'ouverture qui met en communication l'espace (13) et l'oecus (15) dont le montant devait être revêtu de bois, comme cela est suggéré par l'absence du décor - il y a ici seulement une couche préparatoire d'enduit - et par des clous qui, placés à de différentes hauteurs, ont dû servir à fixer les pièces de bois (fig. 13). Le même système est utilisé pour le montant nord de la porte située entre l'espace (13) et la pièce (14), mais seulement sur sa face ouest, celle orientée vers (13). Ici, en effet, le revêtement d'enduit est absent.

Fig. 13. Salon (15), mur sud : détail du revêtement du montant de la porte.

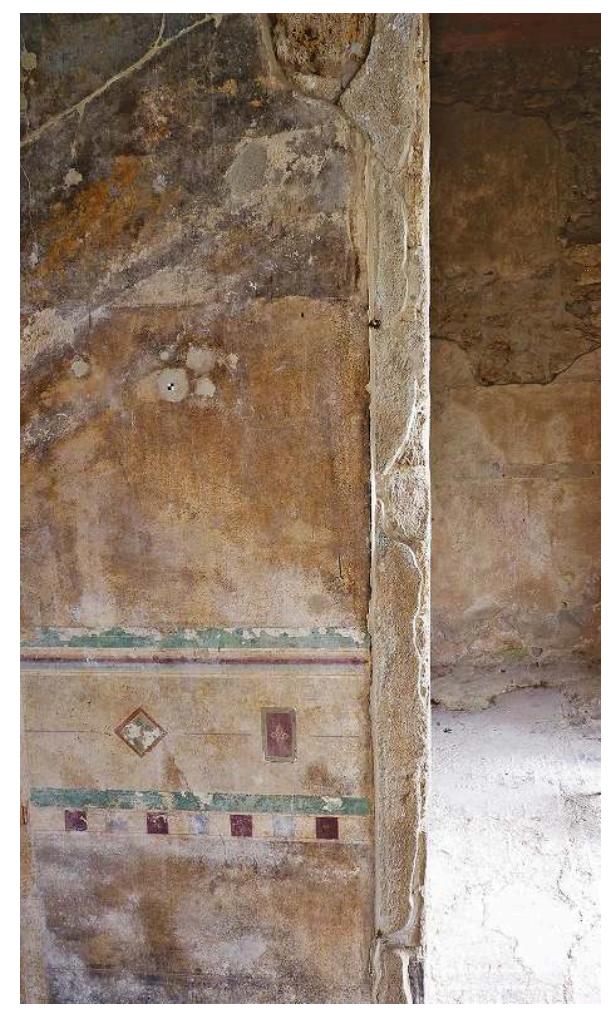

D. D’Auria, sur concession du MiC - PA Pompei. Tous droits réservés.

Les parois de la pièce (13) possédaient également un revêtement, mais son état de conservation ne permet pas d'établir s'il était décoré. Une description synthétique du schéma décoratif des peintures de III ${ }^{\mathrm{e}}$ style des salles (14) et (15) est présentée ci-après.

Les parois de la pièce (14) sont décorées de peintures à fond jaune et à partie basse noire. La composition est centrée, avec une tripartition en trois zones (fig. 14). La zone 1 se compose d'une plinthe surmontée d'une partie basse séparée en trois secteurs par deux rectangles. Chaque secteur est divisé en deux compartiments au moyen d'un filet simple, le long duquel se disposent des formes géométriques: au niveau des panneaux latéraux, un cercle sur la paroi est et des rectangles et un losange sur la paroi nord ; au niveau du panneau central, un carré sur la paroi nord. Dans la zone 1, le décor est enrichi de lignes végétalisées utilisées pour structurer les éléments géométriques et d'un rinceau qui parcourt le panneau central. Deux bandes colorées séparent cette partie de la zone 2 à fond jaune, divisée en trois panneaux et délimitée par une bordure 
verte. Les panneaux latéraux présentent au centre un rectangle et le panneau central comprend un grand tableau à sujet sacro-idyllique. Au-dessus des panneaux, se déploie une frise à fond noir - qu'on reconnaît sur la paroi nord -, figurée et surmontée d'une zone supérieure dans laquelle on reconnaît un pinax, situé dans l'axe du panneau central.

Fig. 14. Pièce (14), paroi est.

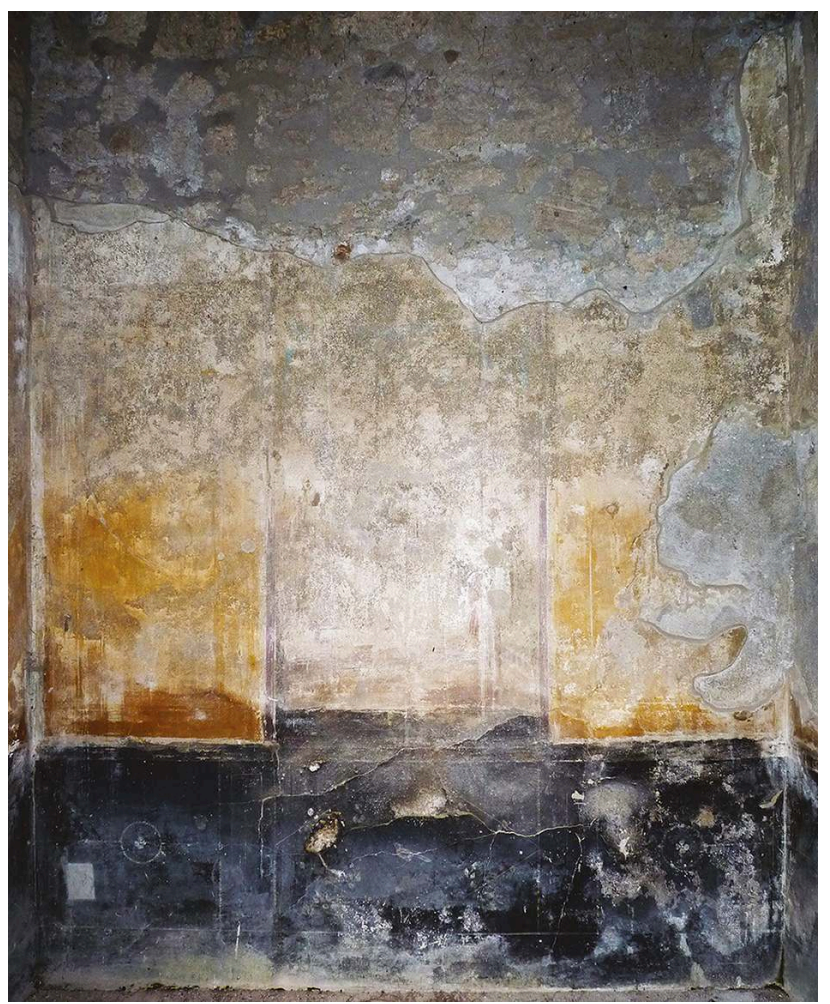

D. D’Auria, sur concession du MiC - PA Pompei. Tous droits réservés.

Les peintures du salon (15), à fond noir, présentent un schéma centré sur un édicule, avec une tripartition en trois zones horizontales (fig. 15). La zone 1 se compose d'une plinthe surmontée d'une partie basse divisée en trois secteurs par les colonnettes de l'édicule central de la zone 2, lesquelles s'appuient sur la plinthe. Chaque secteur est à divisions en croix, au centre desquelles figure un octogone avec un fleuron au milieu. Deux bandes, l'une ornée de carrés en alternance bleu et rouge foncé, l'autre verte, séparent la partie basse d'une prédelle à décor géométrique, composée de rectangles, losanges et carrés polychromes (jaune, vert, rouge foncé). Les colonnettes de l'édicule central séparent les panneaux de la zone 2 et, de plus, les deux panneaux latéraux sont divisés en deux parties par un candélabre reposant sur la base de la prédelle. L'édicule central est occupé au milieu par un tableau à sujet mythologique. L'état de conservation des peintures ne permet pas d'obtenir de données sur les caractéristiques de son entablement, en revanche on distingue la présence d'une frise au-dessus des panneaux. La zone supérieure aussi est dans un mauvais état de conservation, ce qui ne permet pas d'en restituer l'organisation. Le seul élément que nous sommes en mesure de reconnaître est le candélabre de la zone moyenne qui se prolonge jusqu'à ce secteur de la paroi. 
Fig. 15. Le salon (15) vu de l'ouest.

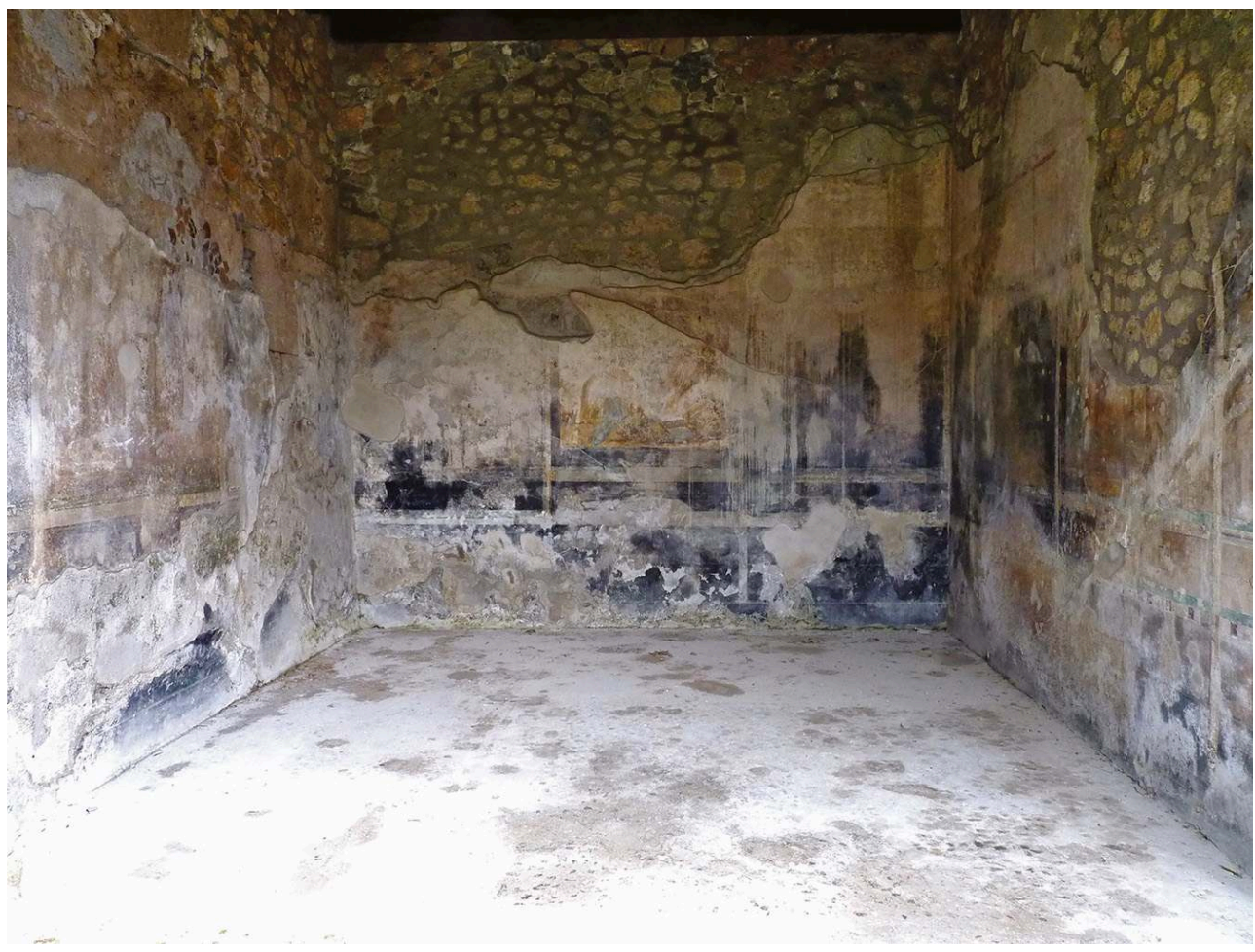

D. D'Auria, sur concession du MiC - PA Pompei. Tous droits réservés par conséquent, elles n'ont pas fait l'objet d'analyse dans le cadre de la campagne photographique et de documentation de Pompéi effectuée par l'Istituto Centrale per il Catalogo e la Documentazione $e^{20}$, qui a débouché sur la publication de Pitture e pavimenti di Pompei: repertorio delle fotografie del Gabinetto Fotografico Nazionale (PPP). Elles sont donc en majeure partie inédites ${ }^{21}$ ce qui constitue un point d'intérêt supplémentaire pour notre recherche. Leur état de conservation n'est pas optimal, certes, les couleurs sont partiellement estompées et il y a des lacunes, parfois de grandes dimensions, comme celles qui concernent les parois sud des deux pièces. Celles-ci, en effet, ont été endommagées par une galerie creusée pendant les premières phases d'exploration de la Regio I. Pour étudier et restituer ces décors, d'un grand intérêt sur le plan stylistique et technique, il a été décidé, au cours de cette première campagne d'études en septembre 2020, d'effectuer un dossier d'imagerie multispectrale, afin de retrouver ces détails qui ne sont désormais plus visibles et d'évaluer la distribution spatiale des pigments employés. Ces techniques - comme l'explique Marcella Leone, infra - ont été particulièrement efficaces pour étudier la polychromie de certains pigments, comme le bleu égyptien.

\section{Phase 4 - Les derniers aménagements avant l'éruption}

Le mur 6 fait l'objet d'une partielle réfection: l'édification des montants en opus vittatum mixtum de la fenêtre et de la porte communiquant avec le portique (8b) (fig. 16). En outre, c'est probablement seulement dans cette phase que le recoin sud de l'espace (13) est transformé en placard : les parois sont revêtues d'un enduit de tuileau, contre lequel sont appuyées deux étagères (ép. $5 \mathrm{~cm}$ env. au centre ; 1,5 cm env. sur les

Bulletin archéologique des Écoles françaises à l'étranger , Italie 
côtés) fixées au mur par des clous et la base est obtenue en étalant une couche de mortier sur la surface du sol de la pièce (13). Le placard était fermé par une porte et profond $55 \mathrm{~cm}$ (fig. 17).

Fig. 16. Salon (15), montant nord de la fenêtre et à droite la structure 1.

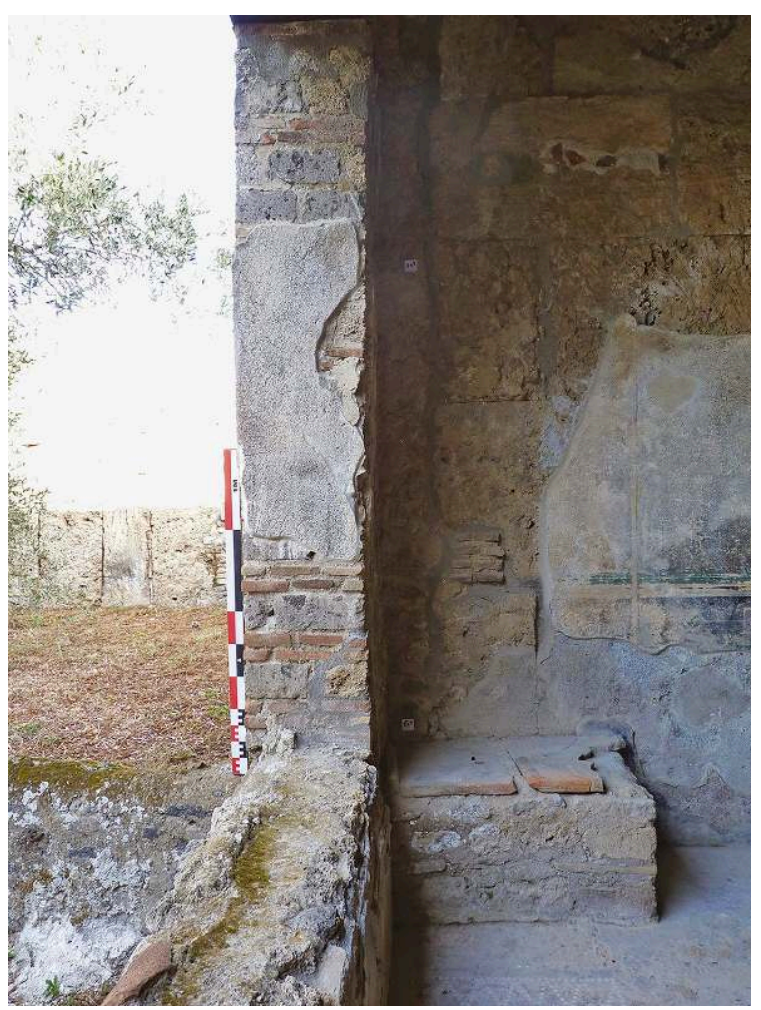

D. D’Auria, sur concession du MiC - PA Pompei. Tous droits réservés. 
Fig. 17. L'armoire de la pièce (13).

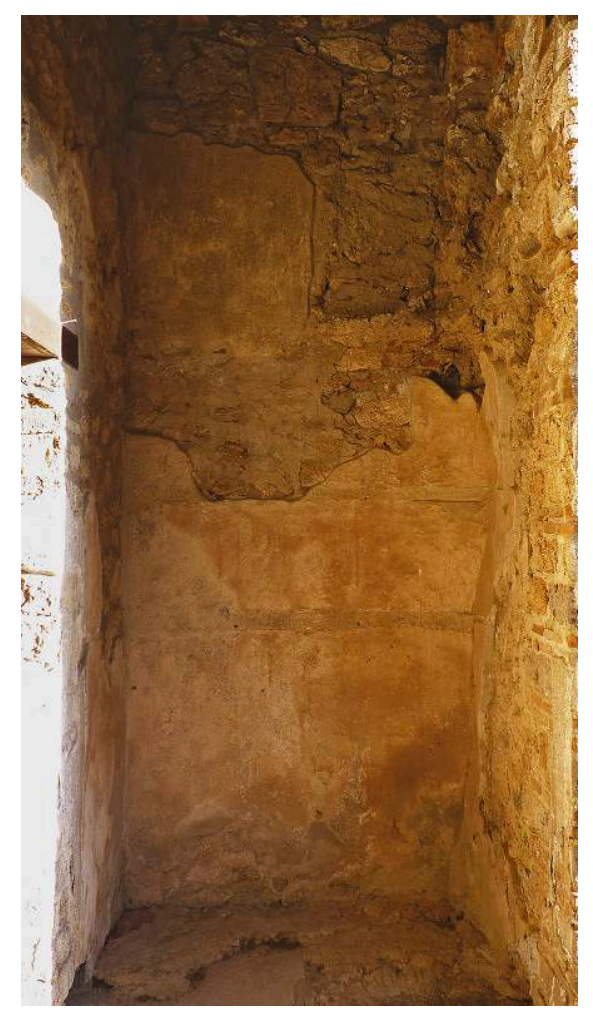

D. D'Auria, sur concession du MiC - PA Pompei. Tous droits réservés.

La dernière intervention discernable à l'intérieur de ces espaces est l'aménagement, en correspondance avec l'angle nord-occidental du salon (15), d'une structure maçonnée (structure 1) de forme rectangulaire, que couvrent deux tuiles (fig. 6 et 16).

\section{Phase 5 - Les pièces (14) et (15) à la veille de l'éruption}

Des données sur l'utilisation de ces pièces les jours précédant l'éruption de 79 apr. J.-C. sont fournies par Salvatore Ciro Nappo, quelques années après la fouille de 1992 (NAPPO 2001, p. 348). L'auteur rapporte que, dans ces pièces, des amphores, des marmites, des outils de travail et des lampes en grand nombre avaient été retrouvés et que, dans la pièce (14), il y avait aussi deux amoncellements de matériel de construction, formant des tas (fig. 18). Il en conclut donc que, à cette époque, la maison 5 était en restructuration. Des détails supplémentaires sur ces travaux ont été fournis par la recherche que nous avons menée dans les archives du Parco Archeologico di Pompei. En effet, dans les journaux de fouille de juin 1959, l'accent est mis sur l'absence de revêtement des parois dans certains secteurs de la maison. C'est le cas de l'atrium, dont les murs sont décrits avec "scarsi rivestimenti d'intonaco signino" le 17 juin et sans enduit le 19. Ces données prennent tout leur sens en les confrontant aux indications fournies par S. C. Nappo, qui nous permettent d'attribuer l'absence de revêtement sur les murs de l'atrium à des travaux qui devaient être en cours dans quelques secteurs de la maison et de savoir que, pendant ces opérations, le matériel de construction et des objets d'usage quotidien avaient été stockés à l'intérieur des pièces (14) et (15). 
Fig. 18. La pièce (14) à l'époque de sa découverte.

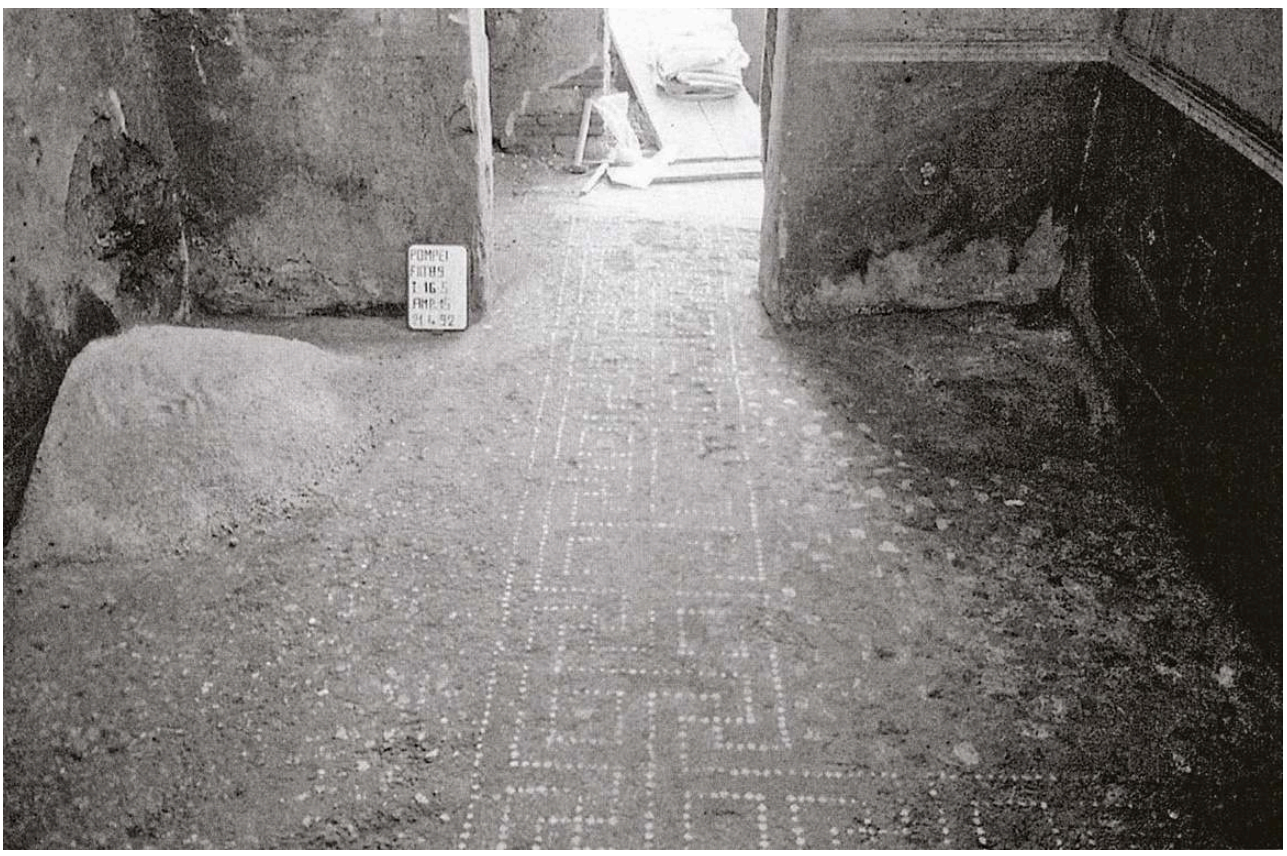

D'après NAPPO 2001.

\section{Imagerie multispectrale, premiers résultats}

M. Leone

Au cours de cette première campagne d'étude, il a été décidé de réaliser un dossier d'imagerie multispectrale. Il s'agit d'un essai d'utilisation de ces méthodes avant d'entreprendre une couverture générale de l'ensemble des parois peintes.

Les techniques photographiques multispectrales, ou multispectral imaging, sont utiles pour une première identification des matériaux et une première caractérisation des pigments, pour définir leur distribution spatiale sur les parois. Les techniques qui ont été utilisées sont la réflectographie infrarouge, la photographie en fluorescence d'ultraviolet et la photographie en luminescence infrarouge ${ }^{22}$. Les images obtenues dans l'infrarouge proche peuvent donner des informations sur la présence de dessins préparatoires et permettre une meilleure lecture des motifs lorsqu'ils ne sont pas très visibles. La fluorescence visible induite par le rayonnement ultraviolet permet de mettre en évidence l'utilisation de matières organiques et, dans une moindre mesure, inorganiques. La photographie en luminescence infrarouge aide à identifier certains pigments comme le bleu égyptien ${ }^{23}$, aussi bien pur que mélangé avec d'autres pigments, ou même lorsqu'il est réduit à l'état de grains invisibles à l'œil nu ${ }^{24}$.

30 La campagne photographique est toujours en cours, mais certains résultats sont déjà appréciables. Nous avons travaillé en particulier sur les parois de la pièce (15).

31 Une première mission photographique a été réalisée en septembre 2020 et effectuée pendant la nuit pour surmonter le problème de la lumière du jour qui rend impossible l'utilisation de certaines techniques ${ }^{25}$.

32 La photographie en fluorescence ultraviolet n'a pas donné de résultats appréciables: aucun élément n'ayant fluorescé, il est donc possible qu'aucun pigment végétal ou laque n'ait été utilisé. 

utilisation du bleu égyptien. Ce pigment artificiel est le seul dans l'Antiquité ; il émet une fluorescence très intense dans l'infrarouge. Il peut être utilisé non seulement pour le bleu, mais aussi en mélange avec une terre verte par exemple, pour créer des nuances de couleur ou pour donner de la luminosité à d'autres couleurs, comme le blanc.

Sur le mur sud de la pièce 15 , le bleu, presque complètement invisible à l'œil nu, est surtout utilisé pour créer des jeux de lumière. Il a été utilisé, par exemple, pour souligner les colonnes qui structurent la composition. Le peintre a souligné le fût de la colonne avec deux lignes bleues qui, semble-t-il, ne sont pas, intentionnellement, de la même épaisseur: l'une est plus épaisse que l'autre, probable stratagème pour augmenter la tridimensionnalité de l'élément, arrondir les colonnes, et le rendre plus lumineux (fig. 19).

Fig. 19. Pièce (15), paroi sud, zone moyenne, détail de la colonne et d'une partie du panneau central.
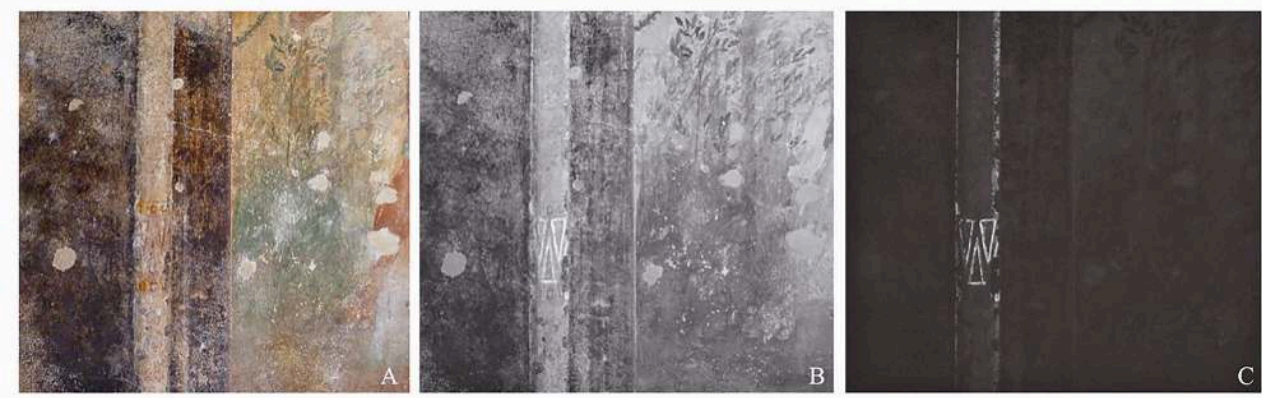

M. Leone, sur concession du MiC - PA Pompei. Tous droits réservés.

Sur la même paroi, à l'intérieur du panneau central de la zone moyenne, le bleu égyptien est également utilisé pour renforcer l'éclat des rouges foncés: la couleur rouge recouvre le pigment bleu, qui est invisible même à une observation attentive (fig. 20).

Fig. 20. Pièce (15), paroi sud, prédelle, panneau central.
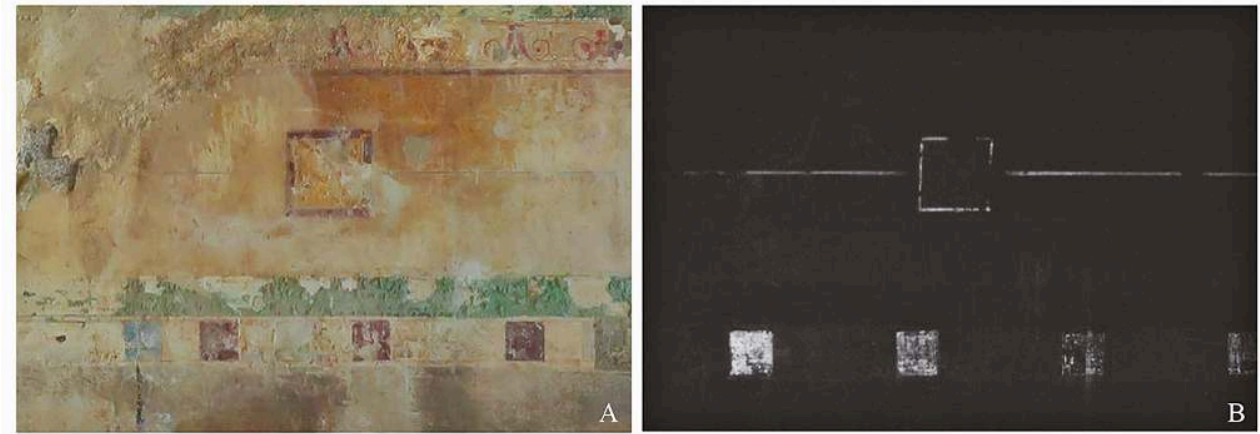

M. Leone, sur concession du MiC - PA Pompei. Tous droits réservés.

Quelques observations intéressantes proviennent également de l'examen des photos dans l'infrarouge proche. Dans le panneau central, la réflectographie infrarouge a mis en évidence les coups de pinceau, surtout visibles dans l'exécution des éléments 
végétaux qui caractérisent la partie gauche du tableau (fig. 19a). Posées sur les larges coups de pinceau verts qui créent un arrière-plan plus homogène, ces touches plus précises et mieux définies créent les détails de la végétation au premier plan (fig. 19b). Pour la couleur verte, l'absence de luminescence induite suggère qu'il ne s'agit pas d'un mélange à base de bleu égyptien. Intéressantes sont aussi les observations que cette technique a permis de faire sur le panneau sud-ouest de la prédelle (fig. 21a). Dans cette zone a été clairement mise en évidence l'utilisation de deux pigments rouges différents, l'un pour la réalisation de la bande rouge bordeaux (corniche fictive supérieure) et des carrés en bas, et l'autre pour le fond du rectangle dressé avec fleuron au centre de ce compartiment (fig. 21b).

Une autre observation qui peut être faite sur les photos en réflectographie infrarouge concerne le fond de ce compartiment de prédelle qui semble être blanc; mais la présence d'une bande blanche, en haut, sous la corniche fictive, et la comparaison avec d'autres zones de la prédelle, laisse supposer que la prédelle était jaune et qu'à cet emplacement le fond a disparu (fig. 21b).

Fig. 21. Pièce (15), paroi sud, prédelle, panneau sud-ouest.
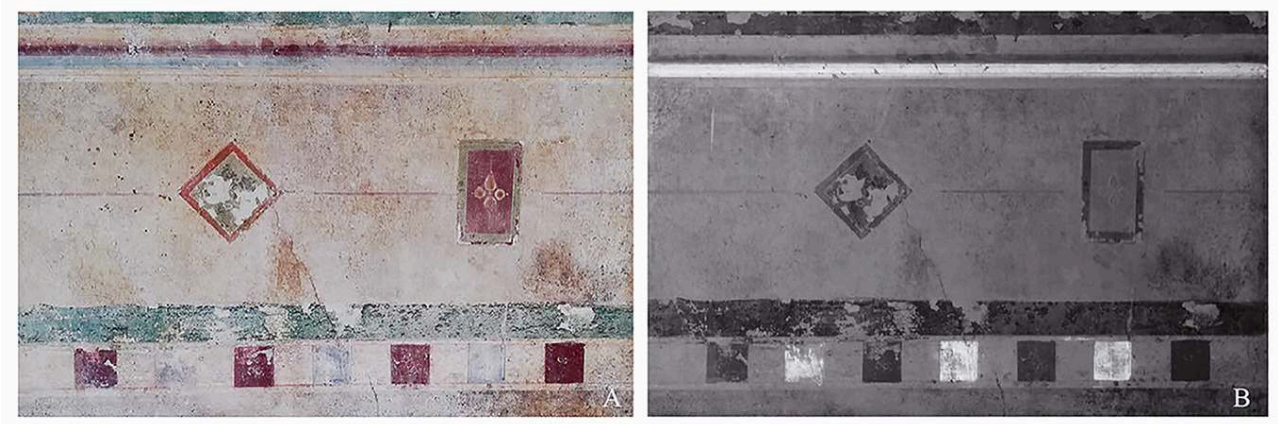

M. Leone, sur concession du MiC - PA Pompei. Tous droits réservés. qui sera dédiée à la réalisation d'un dossier complet en réflectographie infrarouge qui, nous l'espérons, aidera à lire les parties qui ne sont pas clairement visibles.

\section{BIBLIOGRAPHIE}

\section{CAVASSA 2018}

Laetitia Cavassa, « La production du bleu égyptien durant l'époque hellénistique et l'Empire romain (III ${ }^{\mathrm{e}}$ av. J.-C. - Ir ${ }^{\text {er }}$ s. apr. J.-C.) », in Philippe Jockey (dir.), Les arts de la couleur en Grèce ancienne et ailleurs. Approches interdisciplinaires, Athènes, EFA, BCH Suppl. 56, 2018, p. 13-34.

D’AMBROSIO 1993/1994

Antonio D’Ambrosio, « Attività dell'Ufficio Scavi: 1992-1994 », RSP 6-1993/1994, 1994, p. 217-219. 
D'AURIA 2020

Dora D’Auria, Rileggere Pompei VI. Ricerche nella Casa del Granduca Michele (VI,5,5-6/21) e sulle abitazioni di livello medio in età sannitica, Roma, « L'Erma » di Bretschneider, Studi e ricerche del parco archeologico di Pompei 41, 2020.

D'AURIA, BALLET 2020

Dora D’Auria, Pascale Ballet, « Modes d'habiter à Pompéi à l'époque républicaine. Diffusion et utilisation du type de la maison à atrium testudinatum », Chronique des activités archéologiques de l'École française de Rome. Les cités vésuviennes, en ligne, http://journals.openedition.org/cefr/4796, consulté le $1^{\mathrm{er}}$ novembre 2020.

DELAMARE, MONGE, PEPOUX 2004

François Delamare, Gabriel Monge, Monique Pepoux, « À la recherche de différentes qualités marchandes dans les bleus égyptiens trouvés à Pompéi », RSP 15, 2004, p. 89-107.

DE SIMONE, NAPPO 2001

Antonio De Simone, Salvatore Ciro Nappo, « Il completamento dello scavo in alcune insulae delle Regiones I e II », in Pier Giovanni Guzzo (éd.), Pompei. Scienza e società. 250 Anniversario degli Scavi di Pompei, Convegno Internazionale (Napoli, 25-27 novembre 1998), Milan, Electa, 2001, p. 227-228.

DYER, VERRI, CUPITT 2013

Joanne Dyer, Giovanni Verri, John Cupitt, « Multispectral imaging in reflectance and photoinduced luminescence modes: A user manual », Londres 2013, en ligne, https://

research.britishmuseum.org/pdf/charisma-multispectral-imaging-manual-2013.pdf., consulté le 16 janvier 2021

ESCHEBACH 1993

Liselotte Eschebach, Gebäudeverzeichnis und Stadtplan der antiken Stadt Pompeji, Cologne, Böhlau, 1993.

GIULIERINI, CORALINI, SAMPAOLO 2020

Paolo Giulierini, Antonella Coralini, Valeria Sampaolo (éd.), Picta Fragmenta. La pittura vesuviana. Una rilettura. Atti del Convegno Internazionale (Napoli 13-15 settembre 2018), Milan, Silvana Editoriale, 2020.

LAIDLAW 1985

Anne Laidlaw, The First Style in Pompeii: painting and architecture, Rome, G. Bretschneider, 1985.

LAURENZI 2009

Elsa Laurenzi, «Regio XIV, S. Cecilia, domus, (atrio A), Cementizio a base fittile », TESS - fiche 9054-2009, en ligne, http://tess.beniculturali.unipd.it/web/scheda/?recid=9054, consulté le 15 janvier 2020.

MORRICONE MATINI 1971

Maria Luisa Morricone Matini, Pavimenti di signino repubblicani di Roma e dintorni, Rome, Istituto poligrafico dello Stato, Mosaici Antichi in Italia - Studi Monografici 1, 1971.

NAPPO 2001

Salvatore Ciro Nappo, « Nuovi pavimenti in cocciopesto con decorazione geometrica da recenti scavi nella Regio I di Pompei », in Andrea Paribeni (éd.), Atti del VII Colloquio dell'associazione Italiana per lo Studio e la Conservazione del Mosaico (Pompei, 22-25 marzo 2000), Ravenne, Edizioni del Girasole, 2001, p. 343-352. 
PESANDO 1997

Fabrizio Pesando, Domus, edilizia privata e società pompeiana fra III e I secolo a. C., Rome, « L'Erma » di Bretschneider, Monografie 12, 1997.

PIEVE 2013

Paola Pieve, «Varignano, villa sillana, oecus 8, cementizio fittile a inserti e squame », TESS - fiche 14249-2013, en ligne, http://tess.beniculturali.unipd.it/web/scheda/?recid=14249, consulté le 15 janvier 2020.

PPP

Irene Bragantini, Mariette De Vos, Franca Parise Badoni (éd.), Pitture e pavimenti di Pompei: repertorio delle fotografie del Gabinetto Fotografico Nazionale, vol. I-IV, Rome, Ministero per i beni culturali e ambientali, Istituto centrale per il catalogo e la documentazione, 1981-1992.

\section{NOTES}

1. Sur les objectifs du projet et les premières recherches en VI 11, 11-12/7, voir D'AURIA, BALLET 2020.

2. La mission d'étude a été conduite par Pascale Ballet (Université de Paris Nanterre) et Dora D’Auria (Università degli studi di Napoli L'Orientale), avec la collaboration de Guilhem Chapelin (Centre Jean Bérard) qui a réalisé les relevés photogrammétriques, et de Federica Giacobello (Università degli studi di Milano Statale) qui a participé à l'analyse du bâti.

3. La base de données a été élaborée par Guilhem Chapelin et Dora D’Auria.

4. L'ICCD dépend du Ministero della cultura.

5. La documentation produite par nos recherches sera organisée à l'intérieur d'un système d'information construit avec la technologie BIM (Building Information Modeling) qui sera développé en collaboration avec le Centro Interdipartimentale di Servizi di Archeologia (CISA) de l'Università degli studi di Napoli L'Orientale. Il assurera l'intégration des données issues de l'analyse du bâti et des stratigraphies horizontales avec le modèle 3D d'objets paramétriques. Ce système représentera non seulement un outil précieux pour la gestion de la documentation acquise, mais également un support pour la restitution archéologique des contextes analysés.

6. La version manuscrite des mois de mai-décembre 1959 des journaux de fouille n'était pas disponible à la consultation.

7. Ce choix a été motivé par le fait que la date de dégagement de ce secteur de l'îlot I 16, auparavant publiée, était 1958. Cf. ЕSСНЕBACH 1993, 77. Nous remercions Giuseppe Scarpati, responsable des archives du Parco Archeologico di Pompei, pour la disponibilité montrée à notre égard.

8. Ces peintures avaient déjà été vues par LAIDLAW 1985, p. 96.

9. Sur les peintures de la pièce 11, voir D'AURIA 2020, p. 278 et 311-312. Une analyse de la composition des enduits des deux secteurs de la pièce a été effectuée par A. Freccero. Cet examen a confirmé que les deux décorations ont été réalisées en même temps et que leurs enduits ont la même composition que ceux généralement utilisés dans les peintures de ${ }^{\text {er }}$ style, cf. FRECCERO 2012, p. 37-38 et 127-129. 
10. En juin 1959, le secteur à l'ouest de l'atrium n'avait été que partiellement dégagé et apparaissait composé de seuls trois espaces, comme témoigné par le plan à la fig. $\mathbf{2}$.

11. Le péristyle, d'époque samnite, est partiellement démantelé à l'époque impériale. Sur I 16, 2, voir PESANDO 1997, p. 216-217.

12. Le secteur occupé par les pièces (13), (14) et (15) a été mis au jour grâce à des fouilles effectuées en 1992 par la Soprintendenza archeologica di Pompei, avec les fonds FIO et ceux des lois 449 et 64 (D’AMBRosio 1993/1994, p. 218).

13. Cette hypothèse s'appuie sur les données issues de l'examen de la limite occidentale du mur 3 dans la pièce (13-5). En effet, cette partie, comme le mur ouest (mur 6), s'appuie contre un plus ancien mur coupé, qui constituait probablement le premier mur périmétral nord de I 16, 6. Des données supplémentaires, utiles à vérifier cette hypothèse, seront produites par l'analyse stratigraphique des structures de la maison 6 .

14. Également la décoration du tapis, avec un semis irrégulier de grosses écailles polychromes, renvoie à un horizon chronologique de fin $\mathrm{II}^{\mathrm{e}}$ - début $\mathrm{I}^{\mathrm{er}} \mathrm{s}$. av. J.-C. Des sols ayant un type de décoration semblable sont par exemple celui de la domus sous l'église de S. Cecilia à Rome daté du dernier quart du II ${ }^{\mathrm{e}} \mathrm{s}$. av. J.-C. (LAURENZI 2009); celui de l'un des hémicycles de la quatrième terrasse du sanctuaire de Fortuna Primigenia à Préneste, mis en œuvre à l'époque de construction du sanctuaire (MORRICONE MATINI 1971, p. 14 et pl. XIII, n. 51) ou celui de l'oecus (8) de la Villa Sillana de Varignano, daté au premier quart du I ${ }^{\text {er }}$ s. av. J.-C. (PIEVE 2013). Plus de précisions sur la chronologie de ce pavement seront fournies par l'avancement des recherches. Utile à ce propos pourra être, par exemple, l'analyse archéométrique des lithotypes utilisés.

15. Les losanges ont une longueur de $25 / 27 \mathrm{~cm}$ et une largeur de $11 \mathrm{~cm}$.

16. La largeur de la bande est de $13 \mathrm{~cm}$. Les pierres colorées ont une longueur en moyenne de $5 \mathrm{~cm}$.

17. Le tapis est constitué de pierres à la coupe irrégulière avec une longueur qui varie de 2 à $7 \mathrm{~cm}$ et une largeur de 3-4 cm.

18. Les dimensions des pierres sont variables : leur longueur peut aller de 1 à $2,5 \mathrm{~cm}$; leur largeur de 1 à $1,5 \mathrm{~cm}$.

19. La longueur des côtés des tesselles varie de 1,3 à $1,7 \mathrm{~cm}$.

20. Sur cette campagne, qui s'est déroulée de 1977 à 1980, voir Parise Badoni 1981.

21. Dans DE SIMONE, NAPPO 2001, p. 227, ont été publiées deux images des salles (14) et (15) à l'époque de leur découverte, dans lesquelles on voit une partie des peintures. On remarque que les couleurs étaient plus vives en 1992. À l'occasion du colloque international Picta Fragmenta. Rileggendo la pittura vesuviana, qui s'est déroulé à Naples entre le 13 et le 15 septembre 2018, S. C. Nappo a présenté des décorations inédites de $\mathrm{III}^{\mathrm{e}}$ style mises au jour dans la Regio I dans les années 1990. Parmi ces décorations, il y avait les peintures des pièces (14) et (15). Sa contribution n'a pourtant pas fait partie de la publication des actes dans GIULIERINI, CORALINI, SAMPAOLO 2020.

22. Cette technique photographique est connue en anglais comme visible induced infrared luminescence (VIL) ; DYER, VERRI, CUPITT 2013.

23. Il s'agit d'un pigment obtenu par mélange de sable, de cuivre, de calcium et des éléments alcalins; le traitement à haute température de ces éléments permet d'obtenir la cuprorivaïte $\left(\mathrm{CaCuSi}_{4} \mathrm{O}_{10}\right)$, c'est-à-dire le bleu égyptien. Sur la production de ce pigment en Campanie, voir CAVASSA 2018. 
24. Le travail du broyage et donc la taille des grains influe sur la teinte et la luminosité de la couleur : DELAMARE, MONGE, PEPOUX 2004.

25. Les analyses ont été réalisées avec un capteur photographique CCD (système multispectral MADATEC) avec des filtres centrés à $760 \mathrm{~nm}, 850 \mathrm{~nm}$ et $950 \mathrm{~nm}$ et des sources UV LED filtrées.

\section{INDEX}

Thèmes : $\mathrm{CJB}$

peuples https://ark.frantiq.fr/ark:/26678/pcrtS8JHDKUmqH, https://ark.frantiq.fr/ark:/26678/ pcrtkERiWybjc4

chronologie https://ark.frantiq.fr/ark:/26678/pcrtZTmusVUU24

lieux https://ark.frantiq.fr/ark:/26678/pcrt7ya9w00Xhb

Année de l'opération : 2020

sujets https://ark.frantiq.fr/ark:/26678/pcrtWegewfItfX, https://ark.frantiq.fr/ark:/26678/ pcrtBTWy6SwZAu, https://ark.frantiq.fr/ark:/26678/pcrtaodMT8j830

\section{AUTEURS}

\section{DORA D'AURIA}

Università degli studi di Napoli L'Orientale

\section{PASCALE BALLET}

Université Paris Nanterre, UMR 7041 ArScAn - ESPRI

\section{MARCELLA LEONE}

Centre Jean Bérard, USR 3133, CNRS-EFR 